\title{
Finite Unification: Theory and Predictions ${ }^{\star}$
}

\author{
Sven HEINEMEYER ${ }^{\dagger^{1}}$, Myriam MONDRAGÓN ${ }^{\dagger^{2}}$ and George ZOUPANOS ${\dagger^{3} \dagger^{4}}^{4}$ \\ $\dagger^{1}$ Instituto de Física de Cantabria (CSIC-UC), Santander, Spain \\ E-mail: Sven.Heinemeyer@cern.ch \\ $\dagger^{2}$ Instituto de Fúsica, Universidad Nacional Autónoma de México, \\ Apdo. Postal 20-364, México 01000, México \\ E-mail: myriam@fisica.unam.mx \\ $\dagger^{3}$ Theory Group, Physics Department, CERN, Geneva, Switzerland \\ E-mail: George.Zoupanos@cern.ch \\ $\dagger^{4}$ Physics Department, National Technical University, 15780 Zografou, Athens, Greece
}

Received January 03, 2010, in final form May 25, 2010; Published online June 11, 2010

doi:10.3842/SIGMA.2010.049

\begin{abstract}
All-loop Finite Unified Theories (FUTs) are very interesting $N=1$ supersymmetric Grand Unified Theories (GUTs) which not only realise an old field theoretic dream but also have a remarkable predictive power due to the required reduction of couplings. The reduction of the dimensionless couplings in $N=1$ GUTs is achieved by searching for renormalization group invariant (RGI) relations among them holding beyond the unification scale. Finiteness results from the fact that there exist RGI relations among dimensionless couplings that guarantee the vanishing of all beta-functions in certain $N=1$ GUTs even to all orders. Furthermore developments in the soft supersymmetry breaking sector of $N=1$ GUTs and FUTs lead to exact RGI relations, i.e. reduction of couplings, in this dimensionful sector of the theory too. Based on the above theoretical framework phenomenologically consistent FUTS have been constructed. Here we present FUT models based on the $S U(5)$ and $S U(3)^{3}$ gauge groups and their predictions. Of particular interest is the Higgs mass prediction of one of the models which is expected to be tested at the LHC.
\end{abstract}

Key words: unification; gauge theories; finiteness; reduction of couplings

2010 Mathematics Subject Classification: 81T60; 81V22

\section{Introduction}

A large and sustained effort has been done in the recent years aiming to achieve a unified description of all interactions. Out of this endeavor two main directions have emerged as the most promising to attack the problem, namely, the superstring theories and non-commutative geometry. The two approaches, although at a different stage of development, have common unification targets and share similar hopes for exhibiting improved renormalization properties in the ultraviolet (UV) as compared to ordinary field theories. Moreover the two frameworks came closer by the observation that a natural realization of non-commutativity of space appears in the string theory context of D-branes in the presence of a constant background antisymmetric field [1]. Among the numerous important developments in both frameworks, it is worth noting two conjectures of utmost importance that signal the developments in certain directions in string theory and not only, related to the main theme of the present review. The conjectures refer to

* This paper is a contribution to the Proceedings of the Eighth International Conference "Symmetry in Nonlinear Mathematical Physics" (June 21-27, 2009, Kyiv, Ukraine). The full collection is available at http://www.emis.de/journals/SIGMA/symmetry2009.html 
(i) the duality among the 4-dimensional $N=4$ supersymmetric Yang-Mills theory and the type IIB string theory on $\mathrm{AdS}_{5} \times S^{5}$ [2]; the former being the maximal $N=4$ supersymmetric YangMills theory is known to be UV all-loop finite theory [3, 4], (ii) the possibility of "miraculous" UV divergence cancellations in 4-dimensional maximal $N=8$ supergravity leading to a finite theory, as has been recently confirmed in a remarkable 4-loop calculation [5, 6, 7, 8, 9]. However, despite the importance of having frameworks to discuss quantum gravity in a self-consistent way and possibly to construct there finite theories, it is very interesting to search for the minimal realistic framework in which finiteness can take place. In addition, the main goal expected from a unified description of interactions by the particle physics community is to understand the present day large number of free parameters of the Standard Model (SM) in terms of a few fundamental ones. In other words, to achieve reduction of couplings at a more fundamental level.

To reduce the number of free parameters of a theory, and thus render it more predictive, one is usually led to introduce a symmetry. Grand Unified Theories (GUTs) are very good examples of such a procedure $[10,11,12,13,14]$. For instance, in the case of minimal $S U(5)$, because of (approximate) gauge coupling unification, it was possible to reduce the gauge couplings by one and give a prediction for one of them. In fact, LEP data [15] seem to suggest that a further symmetry, namely $N=1$ global supersymmetry $[16,17]$ should also be required to make the prediction viable. GUTs can also relate the Yukawa couplings among themselves, again $S U(5)$ provided an example of this by predicting the ratio $M_{\tau} / M_{b}$ [18] in the SM. Unfortunately, requiring more gauge symmetry does not seem to help, since additional complications are introduced due to new degrees of freedom, in the ways and channels of breaking the symmetry, and so on.

A natural extension of the GUT idea is to find a way to relate the gauge and Yukawa sectors of a theory, that is to achieve gauge-Yukawa Unification (GYU) [19, 20, 21]. A symmetry which naturally relates the two sectors is supersymmetry, in particular $N=2$ supersymmetry [22]. It turns out, however, that $N=2$ supersymmetric theories have serious phenomenological problems due to light mirror fermions. Also in superstring theories and in composite models there exist relations among the gauge and Yukawa couplings, but both kind of theories have phenomenological problems, which we are not going to address here.

There have been other attempts to relate the gauge and Yukawa sectors. One was proposed by Decker, Pestieau, and Veltman [23, 24]. By requiring the absence of quadratic divergences in the SM, they found a relationship between the squared masses appearing in the Yukawa and in the gauge sectors of the theory. A very similar relation is obtained by applying naively in the SM the general formula derived from demanding spontaneous supersymmetry breaking via Fterms [25]. In both cases a prediction for the top quark was possible only when it was permitted experimentally to assume the $M_{H} \ll M_{W, Z}$ with the result $M_{t}=69 \mathrm{GeV}$. Otherwise there is only a quadratic relation among $M_{t}$ and $M_{H}$. Using this relationship in the former case and a version of naturalness into account, i.e. that the quadratic corrections to the Higgs mass be at most equal to the physical mass, the Higgs mass is found to be $\sim 260 \mathrm{GeV}$, for a top quark mass of around $176 \mathrm{GeV}$ [26]. This value is already excluded from the precision data [27].

A well known relation among gauge and Yukawa couplings is the Pendleton-Ross (P-R) infrared fixed point [28]. The P-R proposal, involving the Yukawa coupling of the top quark $g_{t}$ and the strong gauge coupling $\alpha_{3}$, was that the ratio $\alpha_{t} / \alpha_{3}$, where $\alpha_{t}=g_{t}^{2} / 4 \pi$, has an infrared fixed point. This assumption predicted $M_{t} \sim 100 \mathrm{GeV}$. In addition, it has been shown [29] that the P-R conjecture is not justified at two-loops, since then the ratio $\alpha_{t} / \alpha_{3}$ diverges in the infrared.

Another interesting conjecture, made by Hill [30], is that $\alpha_{t}$ itself develops a quasi-infrared fixed point, leading to the prediction $M_{t} \sim 280 \mathrm{GeV}$.

The P-R and Hill conjectures have been done in the framework of the SM. The same conjectures within the Minimal Supersymmetric SM (MSSM) lead to the following relations:

$$
M_{t} \simeq 140 \mathrm{GeV} \sin \beta \quad(\mathrm{P}-\mathrm{R}), \quad M_{t} \simeq 200 \mathrm{GeV} \sin \beta \quad \text { (Hill), }
$$


where $\tan \beta=v_{u} / v_{d}$ is the ratio of the two VEV of the Higgs fields of the MSSM. From theoretical considerations one can expect

$$
1 \lesssim \tan \beta \lesssim 50 \Leftrightarrow \frac{1}{\sqrt{2}} \lesssim \sin \beta \lesssim 1
$$

This corresponds to

$$
100 \mathrm{GeV} \lesssim M_{t} \lesssim 140 \mathrm{GeV} \quad(\mathrm{P}-\mathrm{R}), \quad 140 \mathrm{GeV} \lesssim M_{t} \lesssim 200 \mathrm{GeV} \quad \text { (Hill) }
$$

Thus, the MSSM P-R conjecture is ruled out, while within the MSSM, the Hill conjecture does not give a prediction for $M_{t}$, since the value of $\sin \beta$ is not fixed by other considerations. The Hill model can accommodate the correct value of $M_{t} \approx 173 \mathrm{GeV}$ [31] for $\sin \beta \approx 0.865$ corresponding to $\tan \beta \approx 1$.7. Such small values, however, are stongly challenged by the SUSY Higgs boson searches at LEP [32]. Only a very heavy scalar top spectrum with large mixing could accommodate such a small $\tan \beta$ value.

In our studies [19, 20, 21, 33, 34, 35, 36, 37, 38] we have developed a complementary strategy in searching for a more fundamental theory possibly at the Planck scale, whose basic ingredients are GUTs and supersymmetry, but its consequences certainly go beyond the known ones. Our method consists of hunting for renormalization group invariant (RGI) relations holding below the Planck scale, which in turn are preserved down to the GUT scale. This programme, called gaugeYukawa unification scheme, applied in the dimensionless couplings of supersymmetric GUTs, such as gauge and Yukawa couplings, had already noticable successes by predicting correctly, among others, the top quark mass in the finite and in the minimal $N=1$ supersymmetric $S U(5)$ GUTs [33, 34, 35]. An impressive aspect of the RGI relations is that one can guarantee their validity to all-orders in perturbation theory by studying the uniqueness of the resulting relations at one-loop, as was proven in the early days of the programme of reduction of couplings [39, 40, 41, 42]. Even more remarkable is the fact that it is possible to find RGI relations among couplings that guarantee finiteness to all-orders in perturbation theory [43, 44, 45, 46, 47].

It is worth noting that the above principles have only been applied in supersymmetric GUTs for reasons that will be transparent in the following sections. We should also stress that our conjecture for GYU is by no means in conflict with the interesting proposals mentioned before (see also $[48,49,50]$ ), but it rather uses all of them, hopefully in a more successful perspective. For instance, the use of susy GUTs comprises the demand of the cancellation of quadratic divergences in the SM. Similarly, the very interesting conjectures about the infrared fixed points are generalized in our proposal, since searching for RGI relations among various couplings corresponds to searching for fixed points of the coupled differential equations obeyed by the various couplings of a theory.

Although supersymmetry seems to be an essential feature for a successful realization of the above programme, its breaking has to be understood too, since it has the ambition to supply the SM with predictions for several of its free parameters. Indeed, the search for RGI relations has been extended to the soft supersymmetry breaking sector (SSB) of these theories [38, 51], which involves parameters of dimension one and two. Then a very interesting progress has been made $[52,53,54,55,56,57,58]$ concerning the renormalization properties of the SSB parameters based conceptually and technically on the work of [59]. In [59] the powerful supergraph method $[60,61,62,63]$ for studying supersymmetric theories has been applied to the softly broken ones by using the "spurion" external space-time independent superfields [64]. In the latter method a softly broken supersymmetric gauge theory is considered as a supersymmetric one in which the various parameters such as couplings and masses have been promoted to external superfields that acquire "vacuum expectation values". Based on this method the relations among the soft term renormalization and that of an unbroken supersymmetric theory have been derived. In particular the $\beta$-functions of the parameters of the softly broken theory are expressed in terms 
of partial differential operators involving the dimensionless parameters of the unbroken theory. The key point in the strategy of $[55,56,57,58]$ in solving the set of coupled differential equations so as to be able to express all parameters in a RGI way, was to transform the partial differential operators involved to total derivative operators. This is indeed possible to be done on the RGI surface which is defined by the solution of the reduction equations.

On the phenomenological side there exist some serious developments too. Previously an appealing "universal" set of soft scalar masses was asummed in the SSB sector of supersymmetric theories, given that apart from economy and simplicity (1) they are part of the constraints that preserve finiteness up to two-loops $[65,66]$, (2) they are RGI up to two-loops in more general supersymmetric gauge theories, subject to the condition known as $P=1 / 3 Q$ [51] and (3) they appear in the attractive dilaton dominated supersymmetry breaking superstring scenarios $[67,68,69]$. However, further studies have exhibited a number of problems all due to the restrictive nature of the "universality" assumption for the soft scalar masses. For instance, (a) in finite unified theories the universality predicts that the lightest supersymmetric particle is a charged particle, namely the superpartner of the $\tau$ lepton $\tilde{\tau}$, (b) the MSSM with universal soft scalar masses is inconsistent with the attractive radiative electroweak symmetry breaking [69], and (c) which is the worst of all, the universal soft scalar masses lead to charge and/or colour breaking minima deeper than the standard vacuum [70]. Therefore, there have been attempts to relax this constraint without loosing its attractive features. First an interesting observation was made that in $N=1$ gauge-Yukawa unified theories there exists a RGI sum rule for the soft scalar masses at lower orders; at one-loop for the non-finite case [71] and at two-loops for the finite case [72]. The sum rule manages to overcome the above unpleasant phenomenological consequences. Moreover it was proven [58] that the sum rule for the soft scalar massses is RGI to all-orders for both the general as well as for the finite case. Finally, the exact $\beta$-function for the soft scalar masses in the Novikov-Shifman-Vainstein-Zakharov (NSVZ) scheme [73, 74, 75] for the softly broken supersymmetric QCD has been obtained [58]. Armed with the above tools and results we are in a position to study the spectrum of the full finite models in terms of few free parameters with emphasis on the predictions for the lightest Higgs mass, which is expected to be tested at LHC.

\section{Unification of couplings by the RGI method}

Let us next briefly outline the idea of reduction of couplings. Any RGI relation among couplings (which does not depend on the renormalization scale $\mu$ explicitly) can be expressed, in the implicit form $\Phi\left(g_{1}, \ldots, g_{A}\right)=$ const, which has to satisfy the partial differential equation (PDE)

$$
\mu \frac{d \Phi}{d \mu}=\vec{\nabla} \cdot \vec{\beta}=\sum_{a=1}^{A} \beta_{a} \frac{\partial \Phi}{\partial g_{a}}=0,
$$

where $\beta_{a}$ is the $\beta$-function of $g_{a}$. This PDE is equivalent to a set of ordinary differential equations, the so-called reduction equations (REs) [39, 40, 76],

$$
\beta_{g} \frac{d g_{a}}{d g}=\beta_{a}, \quad a=1, \ldots, A,
$$

where $g$ and $\beta_{g}$ are the primary coupling and its $\beta$-function, and the counting on $a$ does not include $g$. Since maximally $(A-1)$ independent RGI "constraints" in the $A$-dimensional space of couplings can be imposed by the $\Phi_{a}$ 's, one could in principle express all the couplings in terms of a single coupling $g$. The strongest requirement is to demand power series solutions to the REs,

$$
g_{a}=\sum_{n} \rho_{a}^{(n)} g^{2 n+1}
$$


which formally preserve perturbative renormalizability. Remarkably, the uniqueness of such power series solutions can be decided already at the one-loop level [39, 40, 76]. To illustrate this, let us assume that the $\beta$-functions have the form

$$
\begin{aligned}
& \beta_{a}=\frac{1}{16 \pi^{2}}\left[\sum_{b, c, d \neq g} \beta_{a}^{(1) b c d} g_{b} g_{c} g_{d}+\sum_{b \neq g} \beta_{a}^{(1) b} g_{b} g^{2}\right]+\cdots, \\
& \beta_{g}=\frac{1}{16 \pi^{2}} \beta_{g}^{(1)} g^{3}+\cdots,
\end{aligned}
$$

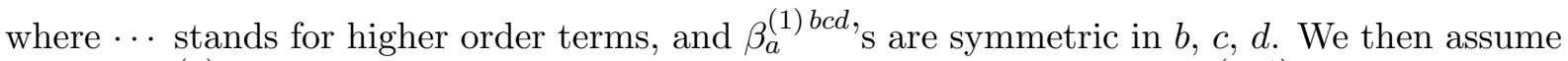
that the $\rho_{a}^{(n)}$ 's with $n \leq r$ have been uniquely determined. To obtain $\rho_{a}^{(r+1)}$, s, we insert the power series (2.2) into the REs (2.1) and collect terms of $\mathcal{O}\left(g^{2 r+3}\right)$ and find

$$
\sum_{d \neq g} M(r)_{a}^{d} \rho_{d}^{(r+1)}=\text { lower order quantities, }
$$

where the r.h.s. is known by assumption, and

$$
\begin{aligned}
& M(r)_{a}^{d}=3 \sum_{b, c \neq g} \beta_{a}^{(1) b c d} \rho_{b}^{(1)} \rho_{c}^{(1)}+\beta_{a}^{(1) d}-(2 r+1) \beta_{g}^{(1)} \delta_{a}^{d}, \\
& 0=\sum_{b, c, d \neq g} \beta_{a}^{(1) b c d} \rho_{b}^{(1)} \rho_{c}^{(1)} \rho_{d}^{(1)}+\sum_{d \neq g} \beta_{a}^{(1) d} \rho_{d}^{(1)}-\beta_{g}^{(1)} \rho_{a}^{(1)} .
\end{aligned}
$$

Therefore, the $\rho_{a}^{(n)}$ 's for all $n>1$ for a given set of $\rho_{a}^{(1)}$ 's can be uniquely determined if $\operatorname{det} M(n)_{a}^{d} \neq 0$ for all $n \geq 0$.

As it will be clear later by examining specific examples, the various couplings in supersymmetric theories have easily the same asymptotic behaviour. Therefore searching for a power series solution of the form (2.2) to the REs (2.1) is justified. This is not the case in non-supersymmetric theories, although the deeper reason for this fact is not fully understood.

The possibility of coupling unification described in this section is without any doubt attractive because the "completely reduced" theory contains only one independent coupling, but it can be unrealistic. Therefore, one often would like to impose fewer RGI constraints, and this is the idea of partial reduction [77, 78].

\section{Reduction of dimensionful parameters}

The reduction of couplings was originally formulated for massless theories on the basis of the Callan-Symanzik equation [39, 40, 76]. The extension to theories with massive parameters is not straightforward if one wants to keep the generality and the rigor on the same level as for the massless case; one has to fulfill a set of requirements coming from the renormalization group equations, the Callan-Symanzik equations, etc. along with the normalization conditions imposed on irreducible Green's functions [79]. See [80] for interesting results in this direction. Here, to simplify the situation, we would like to assume that a mass-independent renormalization scheme has been employed so that all the RG functions have only trivial dependencies of dimensional parameters.

To be general, we consider a renormalizable theory which contain a set of $(N+1)$ dimensionzero couplings, $\left\{\hat{g}_{0}, \hat{g}_{1}, \ldots, \hat{g}_{N}\right\}$, a set of $L$ parameters with dimension one, $\left\{\hat{h}_{1}, \ldots, \hat{h}_{L}\right\}$, and a set of $M$ parameters with dimension two, $\left\{\hat{m}_{1}^{2}, \ldots, \hat{m}_{M}^{2}\right\}$. The renormalized irreducible vertex function satisfies the RG equation

$$
0=\mathcal{D} \Gamma\left[\boldsymbol{\Phi}^{\prime} s ; \hat{g}_{0}, \hat{g}_{1}, \ldots, \hat{g}_{N} ; \hat{h}_{1}, \ldots, \hat{h}_{L} ; \hat{m}_{1}^{2}, \ldots, \hat{m}_{M}^{2} ; \mu\right]
$$




$$
\mathcal{D}=\mu \frac{\partial}{\partial \mu}+\sum_{i=0}^{N} \beta_{i} \frac{\partial}{\partial \hat{g}_{i}}+\sum_{a=1}^{L} \gamma_{a}^{h} \frac{\partial}{\partial \hat{h}_{a}}+\sum_{\alpha=1}^{M} \gamma_{\alpha}^{m^{2}} \frac{\partial}{\partial \hat{m}_{\alpha}^{2}}+\sum_{J} \Phi_{I} \gamma^{\phi I}{ }_{J} \frac{\delta}{\delta \Phi_{J}} .
$$

Since we assume a mass-independent renormalization scheme, the $\gamma$ 's have the form

$$
\begin{aligned}
& \gamma_{a}^{h}=\sum_{b=1}^{L} \gamma_{a}^{h, b}\left(g_{0}, \ldots, g_{N}\right) \hat{h}_{b}, \\
& \gamma_{\alpha}^{m^{2}}=\sum_{\beta=1}^{M} \gamma_{\alpha}^{m^{2}, \beta}\left(g_{0}, \ldots, g_{N}\right) \hat{m}_{\beta}^{2}+\sum_{a, b=1}^{L} \gamma_{\alpha}^{m^{2}, a b}\left(g_{0}, \ldots, g_{N}\right) \hat{h}_{a} \hat{h}_{b},
\end{aligned}
$$

where $\gamma_{a}^{h, b}, \gamma_{\alpha}^{m^{2}, \beta}$ and $\gamma_{a}^{m^{2}, a b}$ are power series of the dimension-zero couplings $g$ 's in perturbation theory.

As in the massless case, we then look for conditions under which the reduction of parameters,

$$
\begin{aligned}
& \hat{g}_{i}=\hat{g}_{i}(g), \quad i=1, \ldots, N, \\
& \hat{h}_{a}=\sum_{b=1}^{P} f_{a}^{b}(g) h_{b}, \quad a=P+1, \ldots, L, \\
& \hat{m}_{\alpha}^{2}=\sum_{\beta=1}^{Q} e_{\alpha}^{\beta}(g) m_{\beta}^{2}+\sum_{a, b=1}^{P} k_{\alpha}^{a b}(g) h_{a} h_{b}, \quad \alpha=Q+1, \ldots, M,
\end{aligned}
$$

is consistent with the RG equation (3.1), where we assume that $g \equiv g_{0}, h_{a} \equiv \hat{h}_{a}(1 \leq a \leq P)$ and $m_{\alpha}^{2} \equiv \hat{m}_{\alpha}^{2}(1 \leq \alpha \leq Q)$ are independent parameters of the reduced theory. We find that the following set of equations has to be satisfied:

$$
\begin{aligned}
& \beta_{g} \frac{\partial \hat{g}_{i}}{\partial g}=\beta_{i}, \quad i=1, \ldots, N, \\
& \beta_{g} \frac{\partial \hat{h}_{a}}{\partial g}+\sum_{b=1}^{P} \gamma_{b}^{h} \frac{\partial \hat{h}_{a}}{\partial h_{b}}=\gamma_{a}^{h}, \quad a=P+1, \ldots, L, \\
& \beta_{g} \frac{\partial \hat{m}_{\alpha}^{2}}{\partial g}+\sum_{a=1}^{P} \gamma_{a}^{h} \frac{\partial \hat{m}_{\alpha}^{2}}{\partial h_{a}}+\sum_{\beta=1}^{Q} \gamma_{\beta}^{m^{2}} \frac{\partial \hat{m}_{\alpha}^{2}}{\partial m_{\beta}^{2}}=\gamma_{\alpha}^{m^{2}}, \quad \alpha=Q+1, \ldots, M .
\end{aligned}
$$

Using equation (3.1) for $\gamma$ 's, one finds that equations (3.5)-(3.7) reduce to

$$
\begin{aligned}
& \beta_{g} \frac{d f_{a}^{b}}{d g}+\sum_{c=1}^{P} f_{a}^{c}\left[\gamma_{c}^{h, b}+\sum_{d=P+1}^{L} \gamma_{c}^{h, d} f_{d}^{b}\right]-\gamma_{a}^{h, b}-\sum_{d=P+1}^{L} \gamma_{a}^{h, d} f_{d}^{b}=0, \\
& a=P+1, \ldots, L, \quad b=1, \ldots, P, \\
& \beta_{g} \frac{d e_{\alpha}^{\beta}}{d g}+\sum_{\gamma=1}^{Q} e_{\alpha}^{\gamma}\left[\gamma_{\gamma}^{m^{2}, \beta}+\sum_{\delta=Q+1}^{M} \gamma_{\gamma}^{m^{2}, \delta} e_{\delta}^{\beta}\right]-\gamma_{\alpha}^{m^{2}, \beta}-\sum_{\delta=Q+1}^{M} \gamma_{\alpha}^{m^{2}, \delta} e_{\delta}^{\beta}=0, \\
& \alpha=Q+1, \ldots, M, \quad \beta=1, \ldots, Q, \\
& \beta_{g} \frac{d k_{\alpha}^{a b}}{d g}+2 \sum_{c=1}^{P}\left(\gamma_{c}^{h, a}+\sum_{d=P+1}^{L} \gamma_{c}^{h, d} f_{d}^{a}\right) k_{\alpha}^{c b}+\sum_{\beta=1}^{Q} e_{\alpha}^{\beta}\left[\gamma_{\beta}^{m^{2}, a b}+\sum_{c, d=P+1}^{L} \gamma_{\beta}^{m^{2}, c d} f_{c}^{a} f_{d}^{b}\right. \\
&\left.+2 \sum_{c=P+1}^{L} \gamma_{\beta}^{m^{2}, c b} f_{c}^{a}+\sum_{\delta=Q+1}^{M} \gamma_{\beta}^{m^{2}, \delta} k_{\delta}^{a b}\right]-\left[\gamma_{\alpha}^{m^{2}, a b}+\sum_{c, d=P+1}^{L} \gamma_{\alpha}^{m^{2}, c d} f_{c}^{a} f_{d}^{b}\right.
\end{aligned}
$$




$$
\begin{aligned}
& \left.+2 \sum_{c=P+1}^{L} \gamma_{\alpha}^{m^{2}, c b} f_{c}^{a}+\sum_{\delta=Q+1}^{M} \gamma_{\alpha}^{m^{2}, \delta} k_{\delta}^{a b}\right]=0, \\
& \alpha=Q+1, \ldots, M, \quad a, b=1, \ldots, P .
\end{aligned}
$$

If these equations are satisfied, the irreducible vertex function of the reduced theory

$$
\begin{aligned}
\Gamma_{R}\left[\boldsymbol{\Phi}^{\prime} s ; g ; h_{1}, \ldots, h_{P} ; m_{1}^{2}, \ldots, \hat{m}_{Q}^{2} ; \mu\right] \\
\equiv \\
\equiv\left[\boldsymbol{\Phi}^{\prime} s ; g, \hat{g}_{1}(g), \ldots, \hat{g}_{N}(g) ; h_{1}, \ldots, h_{P}, \hat{h}_{P+1}(g, h), \ldots, \hat{h}_{L}(g, h) ;\right. \\
\left.\quad m_{1}^{2}, \ldots, \hat{m}_{Q}^{2}, \hat{m}_{Q+1}^{2}\left(g, h, m^{2}\right), \ldots, \hat{m}_{M}^{2}\left(g, h, m^{2}\right) ; \mu\right]
\end{aligned}
$$

has the same renormalization group flow as the original one.

The requirement for the reduced theory to be perturbative renormalizable means that the functions $\hat{g}_{i}, f_{a}^{b}, e_{\alpha}^{\beta}$ and $k_{\alpha}^{a b}$, defined in equations (3.2)-(3.4), should have a power series expansion in the primary coupling $g$ :

$$
\begin{array}{ll}
\hat{g}_{i}=g \sum_{n=0}^{\infty} \rho_{i}^{(n)} g^{n}, & f_{a}^{b}=g \sum_{n=0}^{\infty} \eta_{a}^{b(n)} g^{n}, \\
e_{\alpha}^{\beta}=\sum_{n=0}^{\infty} \xi_{\alpha}^{\beta(n)} g^{n}, & k_{\alpha}^{a b}=\sum_{n=0}^{\infty} \chi_{\alpha}^{a b(n)} g^{n} .
\end{array}
$$

To obtain the expansion coefficients, we insert the power series ansatz above into equations (3.5), (3.8)-(3.10) and require that the equations are satisfied at each order in $g$. Note that the existence of a unique power series solution is a non-trivial matter: It depends on the theory as well as on the choice of the set of independent parameters.

\section{Finiteness in $N=1$ supersymmetric gauge theories}

Let us consider a chiral, anomaly free, $N=1$ globally supersymmetric gauge theory based on a group $G$ with gauge coupling constant $g$. The superpotential of the theory is given by

$$
W=\frac{1}{2} m_{i j} \phi_{i} \phi_{j}+\frac{1}{6} C_{i j k} \phi_{i} \phi_{j} \phi_{k}
$$

where $m_{i j}$ and $C_{i j k}$ are gauge invariant tensors and the matter field $\phi_{i}$ transforms according to the irreducible representation $R_{i}$ of the gauge group $G$. The renormalization constants associated with the superpotential (4.1), assuming that supersymmetry is preserved, are

$$
\begin{aligned}
& \phi_{i}^{0}=\left(Z_{i}^{j}\right)^{(1 / 2)} \phi_{j}, \\
& m_{i j}^{0}=Z_{i j}^{i^{\prime} j^{\prime}} m_{i^{\prime} j^{\prime}}, \\
& C_{i j k}^{0}=Z_{i j k}^{i^{\prime} j^{\prime} k^{\prime}} C_{i^{\prime} j^{\prime} k^{\prime}} .
\end{aligned}
$$

The $N=1$ non-renormalization theorem [81, 82, 62] ensures that there are no mass and cubicinteraction-term infinities and therefore

$$
\begin{aligned}
& Z_{i j k}^{i^{\prime} j^{\prime} k^{\prime}} Z_{i^{\prime}}^{1 / 2 i^{\prime \prime}} Z_{j^{\prime}}^{1 / 2 j^{\prime \prime}} Z_{k^{\prime}}^{1 / 2 k^{\prime \prime}}=\delta_{(i}^{i^{\prime \prime}} \delta_{j}^{j^{\prime \prime}} \delta_{k)}^{k^{\prime \prime}}, \\
& Z_{i j}^{i^{\prime} j^{\prime}} Z_{i^{\prime}}^{1 / 2 i^{\prime \prime}} Z_{j^{\prime}}^{1 / 2 j^{\prime \prime}}=\delta_{(i}^{i^{\prime \prime}} \delta_{j)}^{j^{\prime \prime}} .
\end{aligned}
$$


As a result the only surviving possible infinities are the wave-function renormalization constants $Z_{i}^{j}$, i.e., one infinity for each field. The one-loop $\beta$-function of the gauge coupling $g$ is given by [83]

$$
\beta_{g}^{(1)}=\frac{d g}{d t}=\frac{g^{3}}{16 \pi^{2}}\left[\sum_{i} l\left(R_{i}\right)-3 C_{2}(G)\right],
$$

where $l\left(R_{i}\right)$ is the Dynkin index of $R_{i}$ and $C_{2}(G)$ is the quadratic Casimir of the adjoint representation of the gauge group $G$. The $\beta$-functions of $C_{i j k}$, by virtue of the non-renormalization theorem, are related to the anomalous dimension matrix $\gamma_{i j}$ of the matter fields $\phi_{i}$ as:

$$
\beta_{i j k}=\frac{d C_{i j k}}{d t}=C_{i j l} \gamma_{k}^{l}+C_{i k l} \gamma_{j}^{l}+C_{j k l} \gamma_{i}^{l}
$$

At one-loop level $\gamma_{i j}$ is [83]

$$
\gamma_{j}^{i(1)}=\frac{1}{32 \pi^{2}}\left[C^{i k l} C_{j k l}-2 g^{2} C_{2}\left(R_{i}\right) \delta_{j}^{1}\right]
$$

where $C_{2}\left(R_{i}\right)$ is the quadratic Casimir of the representation $R_{i}$, and $C^{i j k}=C_{i j k}^{*}$. Since dimensional coupling parameters such as masses and couplings of cubic scalar field terms do not influence the asymptotic properties of a theory on which we are interested here, it is sufficient to take into account only the dimensionless supersymmetric couplings such as $g$ and $C_{i j k}$. So we neglect the existence of dimensional parameters, and assume furthermore that $C_{i j k}$ are real so that $C_{i j k}^{2}$ always are positive numbers.

As one can see from equations (4.2) and (4.4), all the one-loop $\beta$-functions of the theory vanish if $\beta_{g}^{(1)}$ and $\gamma_{i j}^{(1)}$ vanish, i.e.

$$
\begin{aligned}
& \sum_{i} \ell\left(R_{i}\right)=3 C_{2}(G), \\
& C^{i k l} C_{j k l}=2 \delta_{j}^{i} g^{2} C_{2}\left(R_{i}\right) .
\end{aligned}
$$

The conditions for finiteness for $N=1$ field theories with $S U(N)$ gauge symmetry are discussed in [84], and the analysis of the anomaly-free and no-charge renormalization requirements for these theories can be found in [85]. A very interesting result is that the conditions (4.5), (4.6) are necessary and sufficient for finiteness at the two-loop level [83, 86, 87, 88, 89].

In case supersymmetry is broken by soft terms, the requirement of finiteness in the one-loop soft breaking terms imposes further constraints among themselves [65]. In addition, the same set of conditions that are sufficient for one-loop finiteness of the soft breaking terms render the soft sector of the theory two-loop finite [65].

The one- and two-loop finiteness conditions (4.5), (4.6) restrict considerably the possible choices of the irreps. $R_{i}$ for a given group $G$ as well as the Yukawa couplings in the superpotential (4.1). Note in particular that the finiteness conditions cannot be applied to the minimal supersymmetric standard model (MSSM), since the presence of a $U(1)$ gauge group is incompatible with the condition $(4.5)$, due to $C_{2}[U(1)]=0$. This naturally leads to the expectation that finiteness should be attained at the grand unified level only, the MSSM being just the corresponding, low-energy, effective theory.

Another important consequence of one- and two-loop finiteness is that supersymmetry (most probably) can only be broken due to the soft breaking terms. Indeed, due to the unacceptability of gauge singlets, F-type spontaneous symmetry breaking [90] terms are incompatible with finiteness, as well as D-type [91] spontaneous breaking which requires the existence of a $U(1)$ gauge group. 
A natural question to ask is what happens at higher loop orders. The answer is contained in a theorem $[44,43]$ which states the necessary and sufficient conditions to achieve finiteness at all orders. Before we discuss the theorem let us make some introductory remarks. The finiteness conditions impose relations between gauge and Yukawa couplings. To require such relations which render the couplings mutually dependent at a given renormalization point is trivial. What is not trivial is to guarantee that relations leading to a reduction of the couplings hold at any renormalization point. As we have seen, the necessary and also sufficient, condition for this to happen is to require that such relations are solutions to the REs

$$
\beta_{g} \frac{d C_{i j k}}{d g}=\beta_{i j k}
$$

and hold at all orders. Remarkably, the existence of all-order power series solutions to (4.7) can be decided at one-loop level, as already mentioned.

Let us now turn to the all-order finiteness theorem $[44,43]$, which states that if a $N=1$ supersymmetric gauge theory can become finite to all orders in the sense of vanishing $\beta$-functions, that is of physical scale invariance. It is based on (a) the structure of the supercurrent in $N=1$ supersymmetric gauge theory [92, 93, 94], and on (b) the non-renormalization properties of $N=1$ chiral anomalies [44, 43, 95, 96, 97]. Details on the proof can be found in [44, 43] and further discussion in $[95,96,97,45,98]$. Here, following mostly [98] we present a comprehensible sketch of the proof.

Consider a $N=1$ supersymmetric gauge theory, with simple Lie group $G$. The content of this theory is given at the classical level by the matter supermultiplets $S_{i}$, which contain a scalar field $\phi_{i}$ and a Weyl spinor $\psi_{i a}$, and the vector supermultiplet $V_{a}$, which contains a gauge vector field $A_{\mu}^{a}$ and a gaugino Weyl spinor $\lambda_{\alpha}^{a}$.

Let us first recall certain facts about the theory:

(1) A massless $N=1$ supersymmetric theory is invariant under a $U(1)$ chiral transformation $R$ under which the various fields transform as follows

$$
\begin{aligned}
& A_{\mu}^{\prime}=A_{\mu}, \quad \lambda_{\alpha}^{\prime}=\exp (-i \theta) \lambda_{\alpha}, \\
& \phi^{\prime}=\exp \left(-i \frac{2}{3} \theta\right) \phi, \quad \psi_{\alpha}^{\prime}=\exp \left(-i \frac{1}{3} \theta\right) \psi_{\alpha},
\end{aligned}
$$

The corresponding axial Noether current $J_{R}^{\mu}(x)$ is

$$
J_{R}^{\mu}(x)=\bar{\lambda} \gamma^{\mu} \gamma^{5} \lambda+\cdots
$$

is conserved classically, while in the quantum case is violated by the axial anomaly

$$
\partial_{\mu} J_{R}^{\mu}=r\left(\epsilon^{\mu \nu \sigma \rho} F_{\mu \nu} F_{\sigma \rho}+\cdots\right) .
$$

From its known topological origin in ordinary gauge theories [99, 100, 101], one would expect that the axial vector current $J_{R}^{\mu}$ to satisfy the Adler-Bardeen theorem and receive corrections only at the one-loop level. Indeed it has been shown that the same non-renormalization theorem holds also in supersymmetric theories [95, 96, 97]. Therefore

$$
r=\hbar \beta_{g}^{(1)} .
$$

(2) The massless theory we consider is scale invariant at the classical level and, in general, there is a scale anomaly due to radiative corrections. The scale anomaly appears in the trace of the energy momentum tensor $T_{\mu \nu}$, which is traceless classically. It has the form

$$
T_{\mu}^{\mu}=\beta_{g} F^{\mu \nu} F_{\mu \nu}+\cdots
$$


(3) Massless, $N=1$ supersymmetric gauge theories are classically invariant under the supersymmetric extension of the conformal group - the superconformal group. Examining the superconformal algebra, it can be seen that the subset of superconformal transformations consisting of translations, supersymmetry transformations, and axial $R$ transformations is closed under supersymmetry, i.e. these transformations form a representation of supersymmetry. It follows that the conserved currents corresponding to these transformations make up a supermultiplet represented by an axial vector superfield called supercurrent $J$,

$$
J \equiv\left\{J_{R}^{\prime \mu}, Q_{\alpha}^{\mu}, T_{\nu}^{\mu}, \ldots\right\}
$$

where $J_{R}^{\prime \mu}$ is the current associated to $R$ invariance, $Q_{\alpha}^{\mu}$ is the one associated to supersymmetry invariance, and $T_{\nu}^{\mu}$ the one associated to translational invariance (energy-momentum tensor).

The anomalies of the $R$ current $J_{R}^{\prime \mu}$, the trace anomalies of the supersymmetry current, and the energy-momentum tensor, form also a second supermultiplet, called the supertrace anomaly

$$
S=\left\{\operatorname{Re} S, \operatorname{Im} S, S_{\alpha}\right\}=\left\{T_{\mu}^{\mu}, \partial_{\mu} J_{R}^{\prime \mu}, \sigma_{\alpha \dot{\beta}}^{\mu} \bar{Q}_{\mu}^{\dot{\beta}}+\cdots\right\},
$$

where $T_{\mu}^{\mu}$ in equation (4.11) and

$$
\partial_{\mu} J_{R}^{\prime \mu}=\beta_{g} \epsilon^{\mu \nu \sigma \rho} F_{\mu \nu} F_{\sigma \rho}+\cdots, \quad \sigma_{\alpha \dot{\beta}}^{\mu} \bar{Q}_{\mu}^{\dot{\beta}}=\beta_{g} \lambda^{\beta} \sigma_{\alpha \beta}^{\mu \nu} F_{\mu \nu}+\cdots .
$$

(4) It is very important to note that the Noether current defined in (4.8) is not the same as the current associated to $R$ invariance that appears in the supercurrent $J$ in (4.12), but they coincide in the tree approximation. So starting from a unique classical Noether current $J_{R \text { (class) }}^{\mu}$, the Noether current $J_{R}^{\mu}$ is defined as the quantum extension of $J_{R \text { (class) }}^{\mu}$ which allows for the validity of the non-renormalization theorem. On the other hand $J_{R}^{\prime \mu}$, is defined to belong to the supercurrent $J$, together with the energy-momentum tensor. The two requirements cannot be fulfilled by a single current operator at the same time.

Although the Noether current $J_{R}^{\mu}$ which obeys (4.9) and the current $J_{R}^{\prime \mu}$ belonging to the supercurrent multiplet $J$ are not the same, there is a relation [44, 43] between quantities associated with them

$$
r=\beta_{g}\left(1+x_{g}\right)+\beta_{i j k} x^{i j k}-\gamma_{A} r^{A},
$$

where $r$ was given in equation (4.10). The $r^{A}$ are the non-renormalized coefficients of the anomalies of the Noether currents associated to the chiral invariances of the superpotential, and - like $r$ - are strictly one-loop quantities. The $\gamma_{A}$ 's are linear combinations of the anomalous dimensions of the matter fields, and $x_{g}$, and $x^{i j k}$ are radiative correction quantities. The structure of equality (4.13) is independent of the renormalization scheme.

One-loop finiteness, i.e. vanishing of the $\beta$-functions at one-loop, implies that the Yukawa couplings $\lambda_{i j k}$ must be functions of the gauge coupling $g$. To find a similar condition to all orders it is necessary and sufficient for the Yukawa couplings to be a formal power series in $g$, which is solution of the REs (4.7).

We can now state the theorem for all-order vanishing $\beta$-functions.

Theorem 1. Consider an $N=1$ supersymmetric Yang-Mills theory, with simple gauge group. If the following conditions are satisfied

1) there is no gauge anomaly;

2) the gauge $\beta$-function vanishes at one-loop

$$
\beta_{g}^{(1)}=0=\sum_{i} l\left(R_{i}\right)-3 C_{2}(G)
$$


3) There exist solutions of the form

$$
C_{i j k}=\rho_{i j k} g, \quad \rho_{i j k} \in \mathbb{C}
$$

to the conditions of vanishing one-loop matter fields anomalous dimensions

$$
\begin{aligned}
\gamma_{j}^{i(1)} & =0 \\
& =\frac{1}{32 \pi^{2}}\left[C^{i k l} C_{j k l}-2 g^{2} C_{2}\left(R_{i}\right) \delta_{i j}\right]
\end{aligned}
$$

4) these solutions are isolated and non-degenerate when considered as solutions of vanishing one-loop Yukawa $\beta$-functions:

$$
\beta_{i j k}=0 \text {. }
$$

Then, each of the solutions (4.14) can be uniquely extended to a formal power series in $g$, and the associated super Yang-Mills models depend on the single coupling constant $g$ with a $\beta$-function which vanishes at all-orders.

It is important to note a few things: The requirement of isolated and non-degenerate solutions guarantees the existence of a unique formal power series solution to the reduction equations. The vanishing of the gauge $\beta$-function at one-loop, $\beta_{g}^{(1)}$, is equivalent to the vanishing of the $R$ current anomaly (4.9). The vanishing of the anomalous dimensions at one-loop implies the vanishing of the Yukawa couplings $\beta$-functions at that order. It also implies the vanishing of the chiral anomaly coefficients $r^{A}$. This last property is a necessary condition for having $\beta$-functions vanishing at all orders ${ }^{1}$.

Proof. Insert $\beta_{i j k}$ as given by the REs into the relationship (4.13) between the axial anomalies coefficients and the $\beta$-functions. Since these chiral anomalies vanish, we get for $\beta_{g}$ an homogeneous equation of the form

$$
0=\beta_{g}(1+O(\hbar))
$$

The solution of this equation in the sense of a formal power series in $\hbar$ is $\beta_{g}=0$, order by order. Therefore, due to the REs (4.7), $\beta_{i j k}=0$ too.

Thus we see that finiteness and reduction of couplings are intimately related. Since an equation like equation (4.13) is lacking in non-supersymmetric theories, one cannot extend the validity of a similar theorem in such theories.

\section{Sum rule for $\mathrm{SB}$ terms in $N=1$ supersymmetric and finite theories: all-loop results}

The method of reducing the dimensionless couplings has been extended [38], to the soft supersymmetry breaking (SSB) dimensionful parameters of $N=1$ supersymmetric theories. In addition it was found [71] that RGI SSB scalar masses in gauge-Yukawa unified models satisfy a universal sum rule. Here we will describe first how the use of the available two-loop RG functions and the requirement of finiteness of the SSB parameters up to this order leads to the soft scalar-mass sum rule [72].

\footnotetext{
${ }^{1}$ There is an alternative way to find finite theories [102].
} 
Consider the superpotential given by (4.1) along with the Lagrangian for SSB terms

$$
-\mathcal{L}_{\mathrm{SB}}=\frac{1}{6} h^{i j k} \phi_{i} \phi_{j} \phi_{k}+\frac{1}{2} b^{i j} \phi_{i} \phi_{j}+\frac{1}{2}\left(m^{2}\right)_{i}^{j} \phi^{* i} \phi_{j}+\frac{1}{2} M \lambda \lambda+\text { h.c. }
$$

where the $\phi_{i}$ are the scalar parts of the chiral superfields $\Phi_{i}, \lambda$ are the gauginos and $M$ their unified mass. Since we would like to consider only finite theories here, we assume that the gauge group is a simple group and the one-loop $\beta$-function of the gauge coupling $g$ vanishes. We also assume that the reduction equations admit power series solutions of the form

$$
C^{i j k}=g \sum_{n} \rho_{(n)}^{i j k} g^{2 n}
$$

According to the finiteness theorem of $[43,44]$, the theory is then finite to all orders in perturbation theory, if, among others, the one-loop anomalous dimensions $\gamma_{i}^{j(1)}$ vanish. The one- and two-loop finiteness for $h^{i j k}$ can be achieved by [66]

$$
h^{i j k}=-M C^{i j k}+\cdots=-M \rho_{(0)}^{i j k} g+O\left(g^{5}\right),
$$

where $\cdots$ stand for higher order terms.

Now, to obtain the two-loop sum rule for soft scalar masses, we assume that the lowest order coefficients $\rho_{(0)}^{i j k}$ and also $\left(m^{2}\right)_{j}^{i}$ satisfy the diagonality relations

$$
\rho_{i p q(0)} \rho_{(0)}^{j p q} \propto \delta_{i}^{j} \quad \text { for all } p \text { and } q \text { and } \quad\left(m^{2}\right)_{j}^{i}=m_{j}^{2} \delta_{j}^{i}
$$

respectively. Then we find the following soft scalar-mass sum rule [72, 21, 103]

$$
\left(m_{i}^{2}+m_{j}^{2}+m_{k}^{2}\right) / M M^{\dagger}=1+\frac{g^{2}}{16 \pi^{2}} \Delta^{(2)}+O\left(g^{4}\right)
$$

for $i, j, k$ with $\rho_{(0)}^{i j k} \neq 0$, where $\Delta^{(2)}$ is the two-loop correction

$$
\Delta^{(2)}=-2 \sum_{l}\left[\left(m_{l}^{2} / M M^{\dagger}\right)-(1 / 3)\right] T\left(R_{l}\right),
$$

which vanishes for the universal choice in accordance with the previous findings [66].

If we know higher-loop $\beta$-functions explicitly, we can follow the same procedure and find higher-loop RGI relations among SSB terms. However, the $\beta$-functions of the soft scalar masses are explicitly known only up to two loops. In order to obtain higher-loop results some relations among $\beta$-functions are needed.

Making use of the spurion technique $[60,61,62,63,64]$, it is possible to find the following all-loop relations among SSB $\beta$-functions, $[52,53,54,55,56,57]$

$$
\begin{aligned}
& \beta_{M}=2 \mathcal{O}\left(\frac{\beta_{g}}{g}\right) \\
& \beta_{h}^{i j k}=\gamma^{i}{ }_{l} h^{l j k}+\gamma^{j}{ }_{l} h^{i l k}+\gamma^{k}{ }_{l} h^{i j l}-2 \gamma_{1 l}^{i} C^{l j k}-2 \gamma_{1 l}^{j} C^{i l k}-2 \gamma_{1}^{k} C^{i j l}, \\
& \left(\beta_{\left.m^{2}\right)^{i}}{ }_{j}=\left[\Delta+X \frac{\partial}{\partial g}\right] \gamma^{i}{ }_{j},\right. \\
& \mathcal{O}=\left(M g^{2} \frac{\partial}{\partial g^{2}}-h^{l m n} \frac{\partial}{\partial C^{l m n}}\right), \\
& \Delta=2 \mathcal{O O}^{*}+2|M|^{2} g^{2} \frac{\partial}{\partial g^{2}}+\tilde{C}_{l m n} \frac{\partial}{\partial C_{l m n}}+\tilde{C}^{l m n} \frac{\partial}{\partial C^{l m n}}
\end{aligned}
$$


where $\left(\gamma_{1}\right)^{i}{ }_{j}=\mathcal{O} \gamma^{i}{ }_{j}, C_{l m n}=\left(C^{l m n}\right)^{*}$, and

$$
\tilde{C}^{i j k}=\left(m^{2}\right)^{i}{ }_{l} C^{l j k}+\left(m^{2}\right)^{j}{ }_{l} C^{i l k}+\left(m^{2}\right)^{k}{ }_{l} C^{i j l} .
$$

It was also found [53] that the relation

$$
h^{i j k}=-M\left(C^{i j k}\right)^{\prime} \equiv-M \frac{d C^{i j k}(g)}{d \ln g},
$$

among couplings is all-loop RGI. Furthermore, using the all-loop gauge $\beta$-function of Novikov et al. $[73,74,75]$ given by

$$
\beta_{g}^{\mathrm{NSVZ}}=\frac{g^{3}}{16 \pi^{2}}\left[\frac{\sum_{l} T\left(R_{l}\right)\left(1-\gamma_{l} / 2\right)-3 C(G)}{1-g^{2} C(G) / 8 \pi^{2}}\right]
$$

it was found the all-loop RGI sum rule [58],

$$
\begin{aligned}
m_{i}^{2}+m_{j}^{2}+m_{k}^{2}= & |M|^{2}\left\{\frac{1}{1-g^{2} C(G) /\left(8 \pi^{2}\right)} \frac{d \ln C^{i j k}}{d \ln g}+\frac{1}{2} \frac{d^{2} \ln C^{i j k}}{d(\ln g)^{2}}\right\} \\
& +\sum_{l} \frac{m_{l}^{2} T\left(R_{l}\right)}{C(G)-8 \pi^{2} / g^{2}} \frac{d \ln C^{i j k}}{d \ln g} .
\end{aligned}
$$

In addition the exact- $\beta$-function for $m^{2}$ in the NSVZ scheme has been obtained [58] for the first time and is given by

$$
\begin{aligned}
\beta_{m_{i}^{2}}^{\mathrm{NSVZ}}= & {\left[|M|^{2}\left\{\frac{1}{1-g^{2} C(G) /\left(8 \pi^{2}\right)} \frac{d}{d \ln g}+\frac{1}{2} \frac{d^{2}}{d(\ln g)^{2}}\right\}\right.} \\
& \left.+\sum_{l} \frac{m_{l}^{2} T\left(R_{l}\right)}{C(G)-8 \pi^{2} / g^{2}} \frac{d}{d \ln g}\right] \gamma_{i}^{\mathrm{NSVZ}} .
\end{aligned}
$$

Surprisingly enough, the all-loop result (5.3) coincides with the superstring result for the finite case in a certain class of orbifold models [72] if $d \ln C^{i j k} / d \ln g=1$.

\section{$6 \quad$ Finite $S U(5)$ Unified Theories}

Finite Unified Theories (FUTs) have always attracted interest for their intriguing mathematical properties and their predictive power. One very important result is that the one-loop finiteness conditions (4.3), (4.4) are sufficient to guarantee two-loop finiteness [83]. A classification of possible one-loop finite models was done by two groups [104, 105, 106]. The first one and two-loop finite $S U(5)$ model was presented in [107], and shortly afterwards the conditions for finiteness in the soft SUSY-breaking sector at one-loop [88] were given. In [108] a one and twoloop finite $S U(5)$ model was presented where the rotation of the Higgs sector was proposed as a way of making it realistic. The first all-loop finite theory was studied in [33, 34], without taking into account the soft breaking terms. Finite soft breaking terms and the proof that one-loop finiteness in the soft terms also implies two-loop finiteness was done in [66]. The inclusion of soft breaking terms in a realistic model was done in [109] and their finiteness to all-loops studied in [56], although the universality of the soft breaking terms lead to a charged LSP. This fact was also noticed in [110], where the inclusion of an extra parameter in the boundary condition of the Higgs mixing mass parameter was introduced to alleviate it. The derivation of the sum-rule in the soft supersymmetry breaking sector and the proof that it can be made all-loop finite were done in [72] and [58] respectively, allowing thus for the construction of all-loop finite realistic models. 
From the classification of theories with vanishing one-loop gauge $\beta$-function [104], one can easily see that there exist only two candidate possibilities to construct $S U(5)$ GUTs with three generations. These possibilities require that the theory should contain as matter fields the chiral supermultiplets $\mathbf{5}, \overline{\mathbf{5}}, \mathbf{1 0}, \overline{\mathbf{5}}, \mathbf{2 4}$ with the multiplicities $(6,9,4,1,0)$ and $(4,7,3,0,1)$, respectively. Only the second one contains a $\mathbf{2 4}$-plet which can be used to provide the spontaneous symmetry breaking (SB) of $S U(5)$ down to $S U(3) \times S U(2) \times U(1)$. For the first model one has to incorporate another way, such as the Wilson flux breaking mechanism to achieve the desired SB of $S U(5)$ $[33,34]$. Therefore, for a self-consistent field theory discussion we would like to concentrate only on the second possibility.

The particle content of the models we will study consists of the following supermultiplets: three $(\overline{\mathbf{5}}+\mathbf{1 0})$, needed for each of the three generations of quarks and leptons, four $(\overline{\mathbf{5}}+\mathbf{5})$ and one $\mathbf{2 4}$ considered as Higgs supermultiplets. When the gauge group of the finite GUT is broken the theory is no longer finite, and we will assume that we are left with the MSSM.

Therefore, a predictive gauge-Yukawa unified $S U(5)$ model which is finite to all orders, in addition to the requirements mentioned already, should also have the following properties:

1. One-loop anomalous dimensions are diagonal, i.e., $\gamma_{i}^{(1) j} \propto \delta_{i}^{j}$.

2. The three fermion generations, in the irreducible representations $\overline{\mathbf{5}}_{i}, \mathbf{1 0}_{i}(i=1,2,3)$, should not couple to the adjoint $\mathbf{2 4}$.

3. The two Higgs doublets of the MSSM should mostly be made out of a pair of Higgs quintet and anti-quintet, which couple to the third generation.

In the following we discuss two versions of the all-order finite model. The model [33, 34], which will be labeled $\mathbf{A}$, and a slight variation of this model (labeled $\mathbf{B}$ ), which can also be obtained from the class of the models suggested in $[54,55]$ with a modification to suppress non-diagonal anomalous dimensions [72].

The superpotential which describes the two models before the reduction of couplings takes places is of the form [33, 34, 72, 107, 108]

$$
\begin{aligned}
W= & \sum_{i=1}^{3}\left[\frac{1}{2} g_{i}^{u} \mathbf{1 0}_{i} \mathbf{1 0}_{i} H_{i}+g_{i}^{d} \mathbf{1 0}_{i} \overline{\mathbf{5}}_{i} \bar{H}_{i}\right]+g_{23}^{u} \mathbf{1 0}_{2} \mathbf{1 0}_{3} H_{4} \\
& +g_{23}^{d} \mathbf{1 0}_{2} \overline{\mathbf{5}}_{3} \bar{H}_{4}+g_{32}^{d} \mathbf{1 0}_{3} \overline{\mathbf{5}}_{2} \bar{H}_{4}+\sum_{a=1}^{4} g_{a}^{f} H_{a} \mathbf{2 4} \bar{H}_{a}+\frac{g^{\lambda}}{3}(\mathbf{2 4})^{3},
\end{aligned}
$$

where $H_{a}$ and $\bar{H}_{a}(a=1, \ldots, 4)$ stand for the Higgs quintets and anti-quintets.

The main difference between model $\mathbf{A}$ and model $\mathbf{B}$ is that two pairs of Higgs quintets and anti-quintets couple to the $\mathbf{2 4}$ in $\mathbf{B}$, so that it is not necessary to mix them with $H_{4}$ and $\bar{H}_{4}$ in order to achieve the triplet-doublet splitting after the symmetry breaking of $S U(5)$ [72]. Thus, although the particle content is the same, the solutions to equations (4.3), (4.4) and the sum rules are different, which will reflect in the phenomenology, as we will see.

\section{$6.1 \quad$ FUTA}

After the reduction of couplings the symmetry of the superpotential $W$ (6.1) is enhanced. For model $\mathbf{A}$ one finds that the superpotential has the $Z_{7} \times Z_{3} \times Z_{2}$ discrete symmetry with the charge assignment as shown in Table 1 , and with the following superpotential

$$
W_{A}=\sum_{i=1}^{3}\left[\frac{1}{2} g_{i}^{u} \mathbf{1 0}_{i} \mathbf{1 0}_{i} H_{i}+g_{i}^{d} \mathbf{1} \mathbf{0}_{i} \overline{\mathbf{s}}_{i} \bar{H}_{i}\right]+g_{4}^{f} H_{4} \mathbf{2 4} \bar{H}_{4}+\frac{g^{\lambda}}{3}(\mathbf{2 4})^{3} .
$$


Table 1. Charges of the $Z_{7} \times Z_{3} \times Z_{2}$ symmetry for model FUTA.

\begin{tabular}{|l|l|l|l|l|l|l|l|l|l|l|l|l|l|l|l|}
\hline & $\overline{\mathbf{5}}_{1}$ & $\overline{\mathbf{5}}_{2}$ & $\overline{\mathbf{5}}_{3}$ & $\mathbf{1 0}_{1}$ & $\mathbf{1 0}_{2}$ & $\mathbf{1 0}_{3}$ & $H_{1}$ & $H_{2}$ & $H_{3}$ & $H_{4}$ & $\bar{H}_{1}$ & $\bar{H}_{2}$ & $\bar{H}_{3}$ & $\bar{H}_{4}$ & $\mathbf{2 4}$ \\
\hline$Z_{7}$ & 4 & 1 & 2 & 1 & 2 & 4 & 5 & 3 & 6 & -5 & -3 & -6 & 0 & 0 & 0 \\
\hline$Z_{3}$ & 0 & 0 & 0 & 1 & 2 & 0 & 1 & 2 & 0 & -1 & -2 & 0 & 0 & 0 & 0 \\
\hline$Z_{2}$ & 1 & 1 & 1 & 1 & 1 & 1 & 0 & 0 & 0 & 0 & 0 & 0 & 0 & 0 & 0 \\
\hline
\end{tabular}

Table 2. Charges of the $Z_{4} \times Z_{4} \times Z_{4}$ symmetry for model FUTB.

\begin{tabular}{|l|l|l|l|l|l|l|l|l|l|l|l|l|l|l|l|}
\hline & $\overline{\mathbf{5}}_{1}$ & $\overline{\mathbf{5}}_{2}$ & $\overline{\mathbf{5}}_{3}$ & $\mathbf{1 0}_{1}$ & $\mathbf{1 0}_{2}$ & $\mathbf{1 0}_{3}$ & $H_{1}$ & $H_{2}$ & $H_{3}$ & $H_{4}$ & $\bar{H}_{1}$ & $\bar{H}_{2}$ & $\bar{H}_{3}$ & $\bar{H}_{4}$ & $\mathbf{2 4}$ \\
\hline$Z_{4}$ & 1 & 0 & 0 & 1 & 0 & 0 & 2 & 0 & 0 & 0 & -2 & 0 & 0 & 0 & 0 \\
\hline$Z_{4}$ & 0 & 1 & 0 & 0 & 1 & 0 & 0 & 2 & 0 & 3 & 0 & -2 & 0 & -3 & 0 \\
\hline$Z_{4}$ & 0 & 0 & 1 & 0 & 0 & 1 & 0 & 0 & 2 & 3 & 0 & 0 & -2 & -3 & 0 \\
\hline
\end{tabular}

The non-degenerate and isolated solutions to $\gamma_{i}^{(1)}=0$ for model FUTA, which are the boundary conditions for the Yukawa couplings at the GUT scale, are:

$$
\begin{aligned}
& \left(g_{1}^{u}\right)^{2}=\frac{8}{5} g^{2}, \quad\left(g_{1}^{d}\right)^{2}=\frac{6}{5} g^{2}, \quad\left(g_{2}^{u}\right)^{2}=\left(g_{3}^{u}\right)^{2}=\frac{8}{5} g^{2}, \\
& \left(g_{2}^{d}\right)^{2}=\left(g_{3}^{d}\right)^{2}=\frac{6}{5} g^{2}, \quad\left(g_{23}^{u}\right)^{2}=0, \quad\left(g_{23}^{d}\right)^{2}=\left(g_{32}^{d}\right)^{2}=0, \\
& \left(g^{\lambda}\right)^{2}=\frac{15}{7} g^{2}, \quad\left(g_{2}^{f}\right)^{2}=\left(g_{3}^{f}\right)^{2}=0, \quad\left(g_{1}^{f}\right)^{2}=0, \quad\left(g_{4}^{f}\right)^{2}=g^{2} .
\end{aligned}
$$

In the dimensionful sector, the sum rule gives us the following boundary conditions at the GUT scale for this model [72]:

$$
m_{H_{u}}^{2}+2 m_{\mathbf{1 0}}^{2}=m_{H_{d}}^{2}+m_{\overline{\mathbf{5}}}^{2}+m_{\mathbf{1 0}}^{2}=M^{2},
$$

and thus we are left with only three free parameters, namely $m_{\overline{\mathbf{5}}} \equiv m_{\overline{\mathbf{5}}_{3}}, m_{\mathbf{1 0}} \equiv m_{\mathbf{1 0}_{3}}$ and $M$.

\subsection{FUTB}

Also in the case of FUTB the symmetry is enhanced after the reduction of couplings. The superpotential has now a $Z_{4} \times Z_{4} \times Z_{4}$ symmetry with charges as shown in Table 2 and with the following superpotential

$$
\begin{aligned}
W_{B}= & \sum_{i=1}^{3}\left[\frac{1}{2} g_{i}^{u} \mathbf{1 0}_{i} \mathbf{1 0} H_{i}+g_{i}^{d} \mathbf{1 0}_{i} \overline{\mathbf{5}}_{i} \bar{H}_{i}\right]+g_{23}^{u} \mathbf{1 0}_{2} \mathbf{1 0}_{3} H_{4} \\
& +g_{23}^{d} \mathbf{1 0}_{2} \overline{\mathbf{5}}_{3} \bar{H}_{4}+g_{32}^{d} \mathbf{1 0}_{3} \overline{\mathbf{5}}_{2} \bar{H}_{4}+g_{2}^{f} H_{2} \mathbf{2 4} \bar{H}_{2}+g_{3}^{f} H_{3} \mathbf{2 4} \bar{H}_{3}+\frac{g^{\lambda}}{3}(\mathbf{2 4})^{3} .
\end{aligned}
$$

For this model the non-degenerate and isolated solutions to $\gamma_{i}^{(1)}=0$ give us:

$$
\begin{aligned}
& \left(g_{1}^{u}\right)^{2}=\frac{8}{5} g^{2}, \quad\left(g_{1}^{d}\right)^{2}=\frac{6}{5} g^{2}, \quad\left(g_{2}^{u}\right)^{2}=\left(g_{3}^{u}\right)^{2}=\frac{4}{5} g^{2}, \\
& \left(g_{2}^{d}\right)^{2}=\left(g_{3}^{d}\right)^{2}=\frac{3}{5} g^{2}, \quad\left(g_{23}^{u}\right)^{2}=\frac{4}{5} g^{2}, \quad\left(g_{23}^{d}\right)^{2}=\left(g_{32}^{d}\right)^{2}=\frac{3}{5} g^{2}, \\
& \left(g^{\lambda}\right)^{2}=\frac{15}{7} g^{2}, \quad\left(g_{2}^{f}\right)^{2}=\left(g_{3}^{f}\right)^{2}=\frac{1}{2} g^{2}, \quad\left(g_{1}^{f}\right)^{2}=0, \quad\left(g_{4}^{f}\right)^{2}=0,
\end{aligned}
$$


and from the sum rule we obtain [72]:

$$
m_{H_{u}}^{2}+2 m_{\mathbf{1 0}}^{2}=M^{2}, \quad m_{H_{d}}^{2}-2 m_{\mathbf{1 0}}^{2}=-\frac{M^{2}}{3}, \quad m_{\overline{\mathbf{5}}}^{2}+3 m_{\mathbf{1 0}}^{2}=\frac{4 M^{2}}{3},
$$

i.e., in this case we have only two free parameters $m_{\mathbf{1 0}} \equiv m_{\mathbf{1 0}_{3}}$ and $M$ for the dimensionful sector.

As already mentioned, after the $S U(5)$ gauge symmetry breaking we assume we have the MSSM, i.e. only two Higgs doublets. This can be achieved by introducing appropriate mass terms that allow to perform a rotation of the Higgs sector [108, 33, 34, 111, 107], in such a way that only one pair of Higgs doublets, coupled mostly to the third family, remains light and acquire vacuum expectation values. To avoid fast proton decay the usual fine tuning to achieve doublet-triplet splitting is performed. Notice that, although similar, the mechanism is not identical to minimal $S U(5)$, since we have an extended Higgs sector.

Thus, after the gauge symmetry of the GUT theory is broken we are left with the MSSM, with the boundary conditions for the third family given by the finiteness conditions, while the other two families are basically decoupled.

We will now examine the phenomenology of such all-loop Finite Unified Theories with $S U(5)$ gauge group and, for the reasons expressed above, we will concentrate only on the third generation of quarks and leptons. An extension to three families, and the generation of quark mixing angles and masses in Finite Unified Theories has been addressed in [112], where several examples are given. These extensions are not considered here.

\subsection{Restrictions from low-energy observables}

Since the gauge symmetry is spontaneously broken below $M_{\mathrm{GUT}}$, the finiteness conditions do not restrict the renormalization properties at low energies, and all it remains are boundary conditions on the gauge and Yukawa couplings (6.2) or (6.3), the $h=-M C$ relation (5.1), and the soft scalar-mass sum rule (5.2) at $M_{\mathrm{GUT}}$, as applied in the two models. Thus we examine the evolution of these parameters according to their RGEs up to two-loops for dimensionless parameters and at one-loop for dimensionful ones with the relevant boundary conditions. Below $M_{\mathrm{GUT}}$ their evolution is assumed to be governed by the MSSM. We further assume a unique supersymmetry breaking scale $M_{\mathrm{SUSY}}$ (which we define as the geometrical average of the stop masses) and therefore below that scale the effective theory is just the SM. This allows to evaluate observables at or below the electroweak scale.

In the following, we briefly describe the low-energy observables used in our analysis. We discuss the current precision of the experimental results and the theoretical predictions. We also give relevant details of the higher-order perturbative corrections that we include. We do not discuss theoretical uncertainties from the RG running between the high-scale parameters and the weak scale. At present, these uncertainties are expected to be less important than the experimental and theoretical uncertainties of the precision observables.

As precision observables we first discuss the 3rd generation quark masses that are leading to the strongest constraints on the models under investigation. Next we apply $B$ physics and Higgs-boson mass constraints. We also briefly discuss the anomalous magnetic moment of the muon.

\subsection{Predictions}

We now present the comparison of the predictions of the four models with the experimental data, see [113] for more details, starting with the heavy quark masses. In Fig. 1 we show the FUTA and FUTB predictions for the top pole mass, $M_{\text {top }}$, and the running bottom mass at the scale $M_{Z}, m_{\text {bot }}\left(M_{Z}\right)$, as a function of the unified gaugino mass $M$, for the two cases $\mu<0$ 

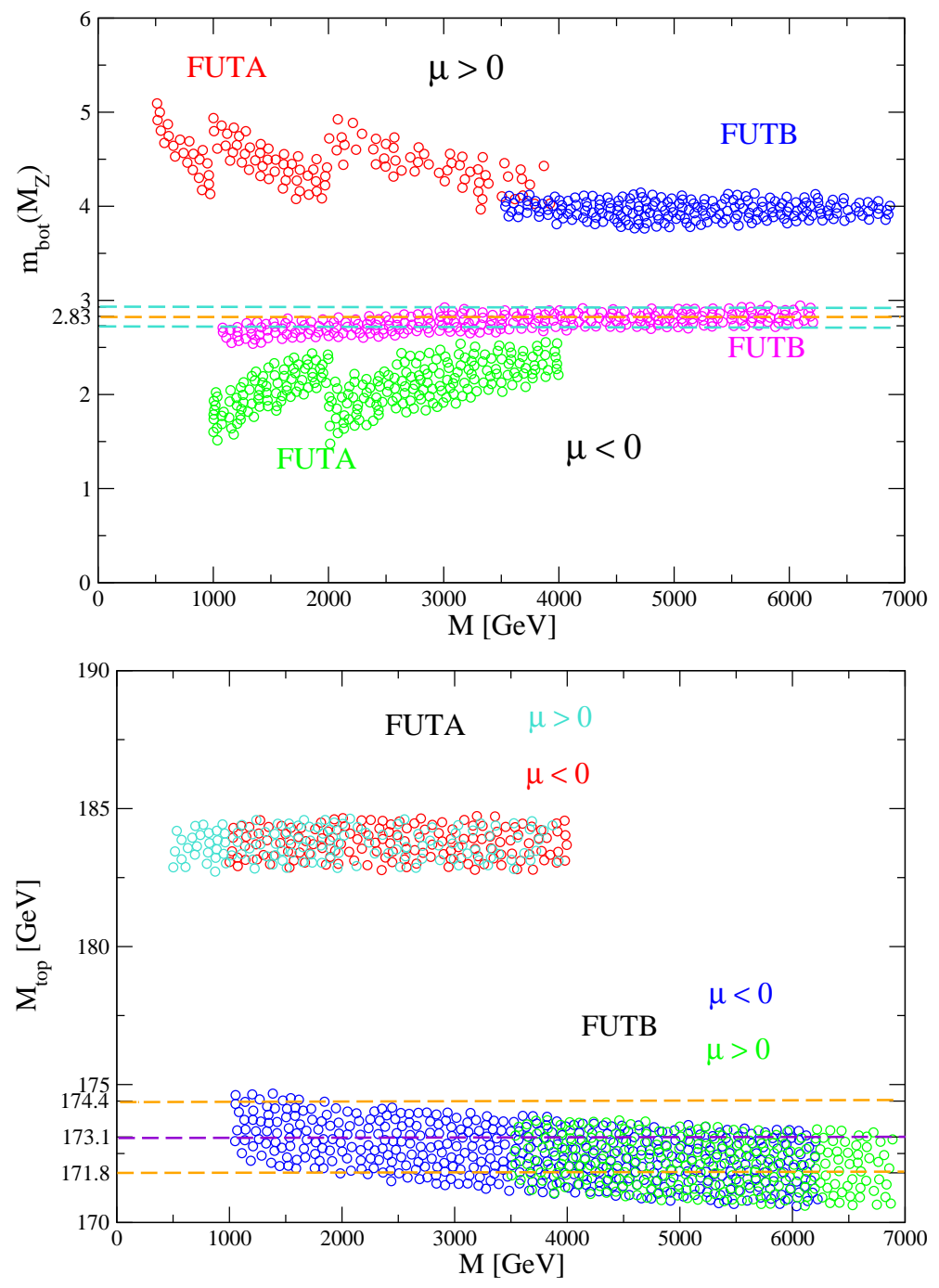

Figure 1. The bottom quark mass at the $Z$ boson scale (upper) and top quark pole mass (lower) are shown as function of $M$ for both models.

and $\mu>0$. The running bottom mass is used to avoid the large QCD uncertainties inherent for the pole mass. In the evaluation of the bottom mass $m_{\text {bot }}$, we have included the corrections coming from bottom squark-gluino loops and top squark-chargino loops [114]. We compare the predictions for the running bottom quark mass with the experimental value, $m_{b}\left(M_{Z}\right)=$ $2.83 \pm 0.10 \mathrm{GeV}[115]$. One can see that the value of $m_{\text {bot }}$ depends strongly on the sign of $\mu$ due to the above mentioned radiative corrections involving SUSY particles. For both models A and $\mathbf{B}$ the values for $\mu>0$ are above the central experimental value, with $m_{\text {bot }}\left(M_{Z}\right) \sim 4.0-$ $5.0 \mathrm{GeV}$. For $\mu<0$, on the other hand, model $\mathbf{B}$ shows overlap with the experimentally measured values, $m_{\text {bot }}\left(M_{Z}\right) \sim 2.5-2.8 \mathrm{GeV}$. For model $\mathbf{A}$ we find $m_{\text {bot }}\left(M_{Z}\right) \sim 1.5-2.6 \mathrm{GeV}$, and there is only a small region of allowed parameter space at large $M$ where we find agreement with the experimental value at the two $\sigma$ level. In summary, the experimental determination of $m_{\text {bot }}\left(M_{Z}\right)$ clearly selects the negative sign of $\mu$.

Now we turn to the top quark mass. The predictions for the top quark mass $M_{\text {top }}$ are $\sim 183$ and $\sim 172 \mathrm{GeV}$ in the models $\mathbf{A}$ and $\mathbf{B}$ respectively, as shown in the lower plot of Fig. 1. Comparing these predictions with the most recent experimental value $m_{t}^{\exp }=(173.1 \pm$ 1.3) $\mathrm{GeV}$ [31], and recalling that the theoretical values for $M_{\text {top }}$ may suffer from a correction of $\sim 4 \%[20,116,103]$, we see that clearly model $\mathbf{B}$ is singled out. In addition the value of $\tan \beta$ is 


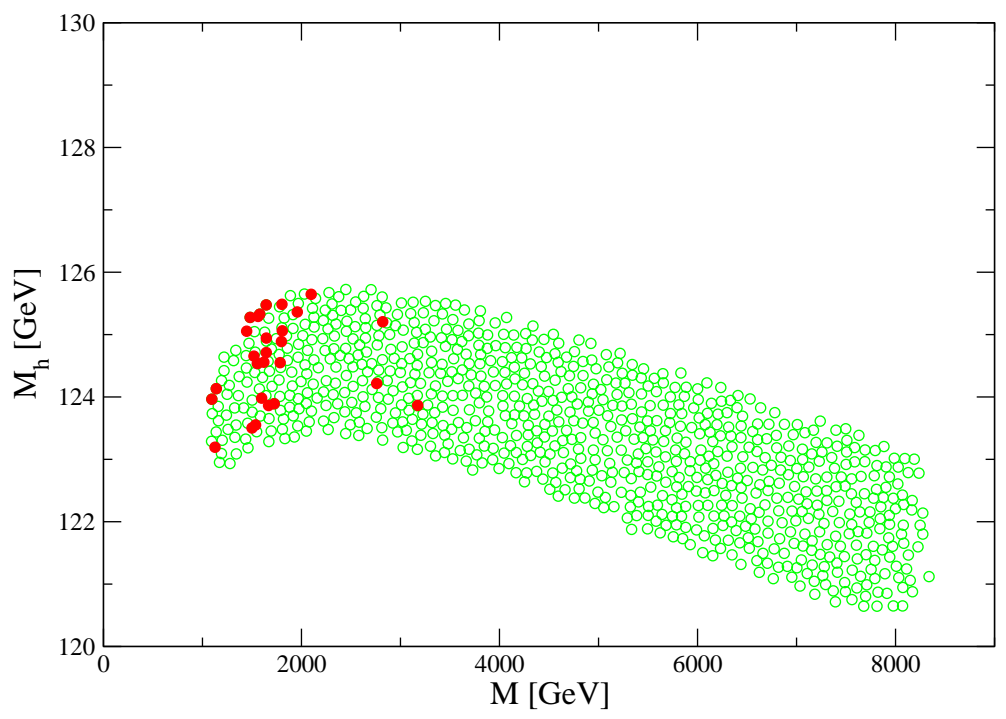

Figure 2. The lightest Higgs mass, $M_{h}$, as function of $M$ for the model FUTB with $\mu<0$, see text.

found to be $\tan \beta \sim 54$ and $\sim 48$ for models $\mathbf{A}$ and $\mathbf{B}$, respectively. Thus from the comparison of the predictions of the two models with experimental data only FUTB with $\mu<0$ survives.

We now analyze the impact of further low-energy observables on the model FUTB with $\mu<0$. As additional constraints we consider the following observables: the rare $b$ decays $\mathrm{BR}(b \rightarrow s \gamma)$ and $\mathrm{BR}\left(B_{s} \rightarrow \mu^{+} \mu^{-}\right)$, the lightest Higgs boson mass as well as the density of cold dark matter in the Universe, assuming it consists mainly of neutralinos. More details and a complete set of references can be found in [113].

For the branching ratio $\mathrm{BR}(b \rightarrow s \gamma)$, we take the experimental value estimated by the Heavy Flavour Averaging Group (HFAG) is [117, 118, 119]

$$
\operatorname{BR}(b \rightarrow s \gamma)=\left(3.55 \pm 0.24_{-0.10}^{+0.09} \pm 0.03\right) \cdot 10^{-4},
$$

where the first error is the combined statistical and uncorrelated systematic uncertainty, the latter two errors are correlated systematic theoretical uncertainties and corrections respectively.

For the branching ratio $\operatorname{BR}\left(B_{s} \rightarrow \mu^{+} \mu^{-}\right)$, the SM prediction is at the level of $10^{-9}$, while the present experimental upper limit from the Tevatron is $4.7 \cdot 10^{-8}$ at the $95 \%$ C.L. [120], still providing the possibility for the MSSM to dominate the SM contribution.

Concerning the lightest Higgs boson mass, $M_{h}$, the SM bound of $114.4 \mathrm{GeV}$ [27, 32] can be applied, since the main SM search channels are not suppressed in FUTB. For the prediction we use the code FeynHiggs [121, 122, 123, 124].

The prediction of the lightest Higgs boson mass as a function of $M$ is shown in Fig. 2. The light (green) points shown are in agreement with the two $B$-physics observables listed above. The lightest Higgs mass ranges in

$$
M_{h} \sim 121-126 \mathrm{GeV},
$$

where the uncertainty comes from variations of the soft scalar masses, and from finite (i.e. not logarithmically divergent) corrections in changing renormalization scheme. To this value one has to add $\pm 3 \mathrm{GeV}$ coming from unknown higher order corrections [123]. We have also included a small variation, due to threshold corrections at the GUT scale, of up to $5 \%$ of the FUT boundary conditions. Thus, taking into account the $B$ physics constraints results naturally in a light Higgs boson that fulfills the LEP bounds [27, 32].

In the same way the whole SUSY particle spectrum can be derived. The resulting SUSY masses for FUTB with $\mu<0$ are rather large. The lightest SUSY particle starts around 
$500 \mathrm{GeV}$, with the rest of the spectrum being very heavy. The observation of SUSY particles at the LHC or the ILC will only be possible in very favorable parts of the parameter space. For most parameter combination only a SM-like light Higgs boson in the range of equation (6.4) can be observed.

We note that with such a heavy SUSY spectrum the anomalous magnetic moment of the muon, $(g-2)_{\mu}$ (with $\left.a_{\mu} \equiv(g-2)_{\mu} / 2\right)$, gives only a negligible correction to the SM prediction. The comparison of the experimental result and the SM value (based on the latest combination using $e^{+} e^{-}$data) [125]

$$
a_{\mu}^{\text {exp }}-a_{\mu}^{\text {theo }}=(24.6 \pm 8.0) \cdot 10^{-10}
$$

would disfavor FUTB with $\mu<0$ by about $3 \sigma$. However, since the SM is not regarded as excluded by $(g-2)_{\mu}$, we still see FUTB with $\mu<0$ as the only surviving model.

Further restrictions on the parameter space can arise from the requirement that the lightest SUSY particle (LSP) should give the right amount of cold dark matter (CDM) abundance. The LSP should be color neutral, and the lightest neutralino appears to be a suitable candidate [126, 127]. In the case where all the soft scalar masses are universal at the unfication scale, there is no region of $M$ below $\mathcal{O}($ few $\mathrm{TeV})$ in which $m_{\tilde{\tau}}>m_{\chi^{0}}$ is satisfied, where $m_{\tilde{\tau}}$ is the lightest $\tilde{\tau}$ mass, and $m_{\chi^{0}}$ the lightest neutralino mass. An electrically charged LSP, however, is not in agreement with CDM searches. But once the universality condition is relaxed this problem can be solved naturally, thanks to the sum rule (5.2). Using this equation a comfortable parameter space is found for FUTB with $\mu<0$ (and also for FUTA and both signs of $\mu$ ). that fulfills the conditions of (a) successful radiative electroweak symmetry breaking, (b) $m_{\tilde{\tau}}>m_{\chi^{0}}$.

Calculating the CDM abundance in these FUT models one finds that usually it is very large, thus a mechanism is needed in our model to reduce it. This issue could, for instance, be related to another problem, that of neutrino masses. This type of masses cannot be generated naturally within the class of finite unified theories that we are considering in this paper, although a nonzero value for neutrino masses has clearly been established [115]. However, the class of FUTs discussed here can, in principle, be easily extended by introducing bilinear $R$-parity violating terms that preserve finiteness and introduce the desired neutrino masses [128]. $R$-parity violation $[129,130,131,132]$ would have a small impact on the collider phenomenology discussed here (apart from fact the SUSY search strategies could not rely on a "missing energy" signature), but remove the CDM bound completely. The details of such a possibility in the present framework attempting to provide the models with realistic neutrino masses will be discussed elsewhere. Other mechanisms, not involving $R$-parity violation (and keeping the "missing energy" signature), that could be invoked if the amount of CDM appears to be too large, concern the cosmology of the early universe. For instance, "thermal inflation" [133] or "late time entropy injection" [134] could bring the CDM density into agreement with the WMAP measurements. This kind of modifications of the physics scenario neither concerns the theory basis nor the collider phenomenology, but could have a strong impact on the CDM derived bounds.

Therefore, in order to get an impression of the possible impact of the CDM abundance on the collider phenomenology in our models under investigation, we will analyze the case that the LSP does contribute to the CDM density, and apply a more loose bound of

$$
\Omega_{\mathrm{CDM}} h^{2}<0.3
$$

(Lower values than the ones permitted by (6.5) are naturally allowed if another particle than the lightest neutralino constitutes CDM.) For our evaluation we have used the code MicroMegas [135, 136]. The prediction for the lightest Higgs mass, $M_{h}$ as function of $M$ for the model FUTB with $\mu<0$ is shown in Fig. 2. The dark (red) dots are the points that pass the constraints in (6.5) (and that have the lightest neutralino as LSP), which favors relatively light values of $M$. The full 


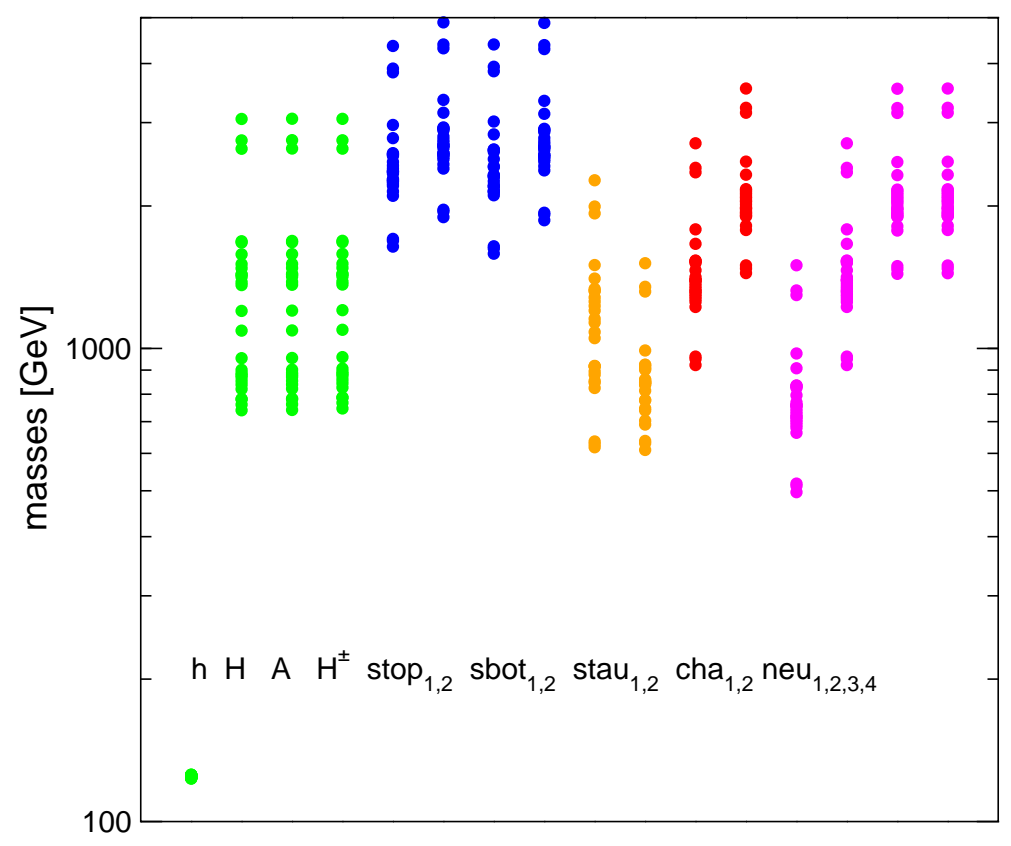

Figure 3. The particle spectrum of model FUTB with $\mu<0$, where the points shown are in agreement with the quark mass constraints, the $B$-physics observables and the loose CDM constraint. The light (green) points on the left are the various Higgs boson masses. The dark (blue) points following are the two scalar top and bottom masses, followed by lighter (beige) scalar tau masses. The darker (red) points to the right are the two chargino masses followed by the lighter shaded (pink) points indicating the neutralino masses.

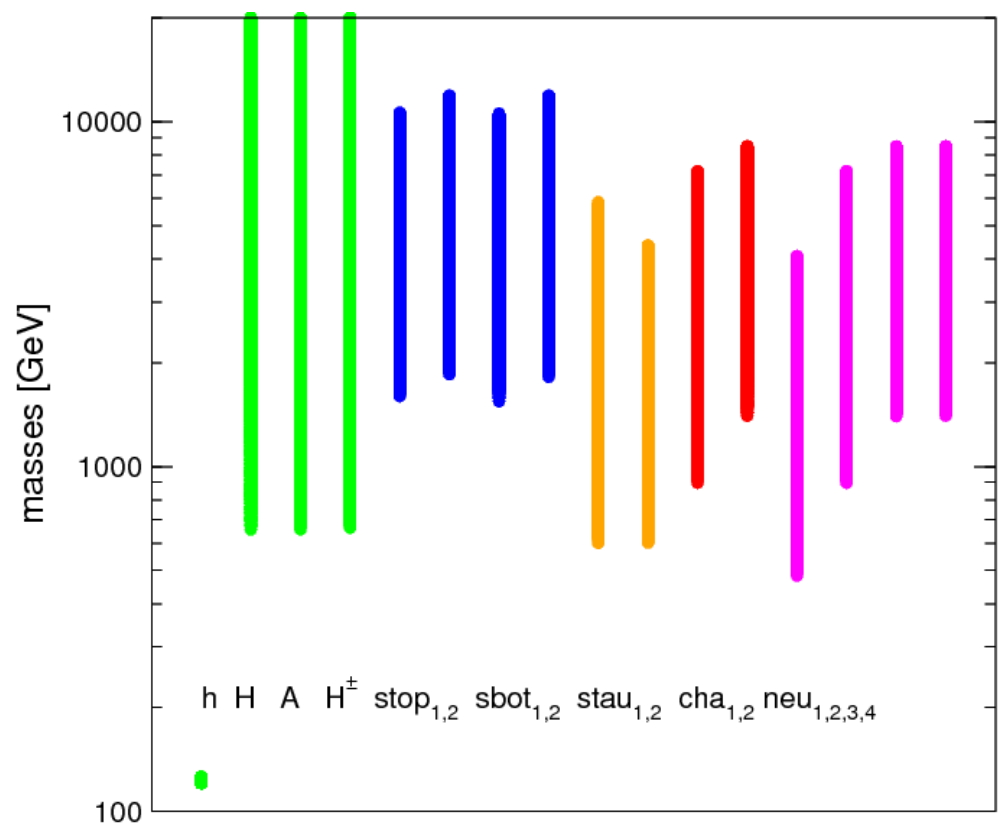

Figure 4. The particle spectrum of model FUTB with $\mu<0$, the points shown are in agreement with the quark mass constraints, the $B$-physics observables, but the loose CDM constraint has been omitted (motivated by possible $R$-parity violation, see text). The color coding is as in Fig. 3.

particle spectrum of model FUTB with $\mu<0$, again compliant with quark mass constraints, the $B$ physics observables (and with the loose CDM constraint), is shown in Fig. 3. The masses of the particles increase with increasing values of the unified gaugino mass $M$. One can see that 
Table 3. A representative spectrum of a light FUTB, $\mu<0$ spectrum.

\begin{tabular}{|l|l|l|l|}
\hline Mbot $\left(M_{Z}\right)$ & $2.71 \mathrm{GeV}$ & Mtop & $172.2 \mathrm{GeV}$ \\
\hline Mh & $123.1 \mathrm{GeV}$ & MA & $680 \mathrm{GeV}$ \\
\hline MH & $679 \mathrm{GeV}$ & MH$^{ \pm}$ & $685 \mathrm{GeV}$ \\
\hline Stop1 & $1876 \mathrm{GeV}$ & Stop2 & $2146 \mathrm{GeV}$ \\
\hline Sbot1 & $1849 \mathrm{GeV}$ & Sbot2 & $2117 \mathrm{GeV}$ \\
\hline Mstau1 & $635 \mathrm{GeV}$ & Mstau2 & $867 \mathrm{GeV}$ \\
\hline Char1 & $1072 \mathrm{GeV}$ & Char2 & $1597 \mathrm{GeV}$ \\
\hline Neu1 & $579 \mathrm{GeV}$ & Neu2 & $1072 \mathrm{GeV}$ \\
\hline Neu3 & $1591 \mathrm{GeV}$ & Neu4 & $1596 \mathrm{GeV}$ \\
\hline M1 & $580 \mathrm{GeV}$ & M2 & $1077 \mathrm{GeV}$ \\
\hline Mgluino & $2754 \mathrm{GeV}$ & & \\
\hline
\end{tabular}

large parts of the spectrum are in the kinematic reach of the LHC. A numerical example of such a light spectrum is shown in Table 3. The colored part of this spectrum as well as the lightest Higgs boson should be (relatively easily) accessible at the LHC.

Finally for the model FUTB with $\mu<0$ we show the particle spectrum, where only the quark mass constraints and the $B$ physics observables are taken into account. The loose CDM constraint, on the other hand, has been omitted, motivated by the possible $R$-parity violation as discussed above. Consequently, following Fig. 2, larger values of $M$ are allowed, resulting in a heavier spectrum, as can be seen in Fig. 4. In this case only part of the parameter space would be in the kinematic reach of the LHC. Only the SM-like light Higgs boson remains observable for the whole parameter range.

A more detailed analysis can be found in [113].

\section{$7 \quad$ Finite $S U(3)^{3}$ model}

We now examine the possibility of constructing realistic FUTs based on product gauge groups. Consider an $N=1$ supersymmetric theory, with gauge group $S U(N)_{1} \times S U(N)_{2} \times \cdots \times$ $S U(N)_{k}$, with $n_{f}$ copies (number of families) of the supersymmetric multiplets $\left(N, N^{*}, 1, \ldots, 1\right)+$ $\left(1, N, N^{*}, \ldots, 1\right)+\cdots+\left(N^{*}, 1,1, \ldots, N\right)$. The one-loop $\beta$-function coefficient in the renormalization-group equation of each $S U(N)$ gauge coupling is simply given by

$$
b=\left(-\frac{11}{3}+\frac{2}{3}\right) N+n_{f}\left(\frac{2}{3}+\frac{1}{3}\right)\left(\frac{1}{2}\right) 2 N=-3 N+n_{f} N .
$$

This means that $n_{f}=3$ is the only solution of equation (7.1) that yields $b=0$. Since $b=0$ is a necessary condition for a finite field theory, the existence of three families of quarks and leptons is natural in such models, provided the matter content is exactly as given above.

The model of this type with best phenomenology is the $S U(3)^{3}$ model discussed in [137], where the details of the model are given. It corresponds to the well-known example of $S U(3)_{C} \times$ $S U(3)_{L} \times S U(3)_{R}[138,139,140,141]$, with quarks transforming as

$$
q=\left(\begin{array}{lll}
d & u & h \\
d & u & h \\
d & u & h
\end{array}\right) \sim\left(3,3^{*}, 1\right), \quad q^{c}=\left(\begin{array}{lll}
d^{c} & d^{c} & d^{c} \\
u^{c} & u^{c} & u^{c} \\
h^{c} & h^{c} & h^{c}
\end{array}\right) \sim\left(3^{*}, 1,3\right),
$$

and leptons transforming as

$$
\lambda=\left(\begin{array}{ccc}
N & E^{c} & \nu \\
E & N^{c} & e \\
\nu^{c} & e^{c} & S
\end{array}\right) \sim\left(1,3,3^{*}\right)
$$


Switching the first and third rows of $q^{c}$ together with the first and third columns of $\lambda$, we obtain the alternative left-right model first proposed in [141] in the context of superstring-inspired $E_{6}$.

In order for all the gauge couplings to be equal at $M_{\mathrm{GUT}}$, as is suggested by the LEP results [15], the cyclic symmetry $Z_{3}$ must be imposed, i.e.

$$
q \rightarrow \lambda \rightarrow q^{c} \rightarrow q
$$

where $q$ and $q^{c}$ are given in equation (7.2) and $\lambda$ in equation (7.3). Then, the first of the finiteness conditions (4.5) for one-loop finiteness, namely the vanishing of the gauge $\beta$-function is satisfied.

Next let us consider the second condition, i.e. the vanishing of the anomalous dimensions of all superfields, equation (4.6). To do that first we have to write down the superpotential. If there is just one family, then there are only two trilinear invariants, which can be constructed respecting the symmetries of the theory, and therefore can be used in the superpotential as follows

$$
f \operatorname{Tr}\left(\lambda q^{c} q\right)+\frac{1}{6} f^{\prime} \epsilon_{i j k} \epsilon_{a b c}\left(\lambda_{i a} \lambda_{j b} \lambda_{k c}+q_{i a}^{c} q_{j b}^{c} q_{k c}^{c}+q_{i a} q_{j b} q_{k c}\right),
$$

where $f$ and $f^{\prime}$ are the Yukawa couplings associated to each invariant. Quark and leptons obtain masses when the scalar parts of the superfields $\left(\tilde{N}, \tilde{N}^{c}\right)$ obtain vacuum expectation values (vevs),

$$
m_{d}=f\langle\tilde{N}\rangle, \quad m_{u}=f\left\langle\tilde{N}^{c}\right\rangle, \quad m_{e}=f^{\prime}\langle\tilde{N}\rangle, \quad m_{\nu}=f^{\prime}\left\langle\tilde{N}^{c}\right\rangle .
$$

With three families, the most general superpotential contains $11 f$ couplings, and $10 f^{\prime}$ couplings, subject to 9 conditions, due to the vanishing of the anomalous dimensions of each superfield. The conditions are the following

$$
\sum_{j, k} f_{i j k}\left(f_{l j k}\right)^{*}+\frac{2}{3} \sum_{j, k} f_{i j k}^{\prime}\left(f_{l j k}^{\prime}\right)^{*}=\frac{16}{9} g^{2} \delta_{i l},
$$

where

$$
f_{i j k}=f_{j k i}=f_{k i j}, \quad f_{i j k}^{\prime}=f_{j k i}^{\prime}=f_{k i j}^{\prime}=f_{i k j}^{\prime}=f_{k j i}^{\prime}=f_{j i k}^{\prime}
$$

Quarks and leptons receive masses when the scalar part of the superfields $\tilde{N}_{1,2,3}$ and $\tilde{N}_{1,2,3}^{c}$ obtain vevs as follows

$$
\begin{array}{rlrl}
\left(\mathcal{M}_{d}\right)_{i j} & =\sum_{k} f_{k i j}\left\langle\tilde{N}_{k}\right\rangle, & \left(\mathcal{M}_{u}\right)_{i j}=\sum_{k} f_{k i j}\left\langle\tilde{N}_{k}^{c}\right\rangle, \\
\left(\mathcal{M}_{e}\right)_{i j}=\sum_{k} f_{k i j}^{\prime}\left\langle\tilde{N}_{k}\right\rangle, & \left(\mathcal{M}_{\nu}\right)_{i j}=\sum_{k} f_{k i j}^{\prime}\left\langle\tilde{N}_{k}^{c}\right\rangle .
\end{array}
$$

We will assume that the below $M_{\mathrm{GUT}}$ we have the usual MSSM, with the two Higgs doublets coupled maximally to the third generation. Therefore we have to choose the linear combinations $\tilde{N}^{c}=\sum_{i} a_{i} \tilde{N}_{i}^{c}$ and $\tilde{N}=\sum_{i} b_{i} \tilde{N}_{i}$ to play the role of the two Higgs doublets, which will be responsible for the electroweak symmetry breaking. This can be done by choosing appropriately the masses in the superpotential [108], since they are not constrained by the finiteness conditions. We choose that the two Higgs doublets are predominately coupled to the third generation. Then these two Higgs doublets couple to the three families differently, thus providing the freedom to understand their different masses and mixings. The remnants of the $S U(3)^{3} \mathrm{FUT}$ are the boundary conditions on the gauge and Yukawa couplings, i.e. (7.4), the $h=-M C$ relation, 
and the soft scalar-mass sum rule equation (5.2) at $M_{\mathrm{GUT}}$, which, when applied to the present model, takes the form

$$
m_{H_{u}}^{2}+m_{\tilde{t}^{c}}^{2}+m_{\tilde{q}}^{2}=M^{2}=m_{H_{d}}^{2}+m_{\tilde{b}^{c}}^{2}+m_{\tilde{q}}^{2},
$$

where $\tilde{t}^{c}, \tilde{b}^{c}$, and $\tilde{q}$ are the scalar parts of the corresponding

Concerning the solution to equation (7.4) we consider two versions of the model:

I) An all-loop finite model with a unique and isolated solution, in which $f^{\prime}$ vanishes, which leads to the following relation

$$
f^{2}=f_{111}^{2}=f_{222}^{2}=f_{333}^{2}=\frac{16}{9} g^{2} .
$$

As for the lepton masses, because all $f^{\prime}$ couplings have been fixed to be zero at this order, in principle they would be expected to appear radiatively induced by the scalar lepton masses appearing in the SSB sector of the theory. However, due to the finiteness conditions they cannot appear radiatively and remain as a problem for further study.

II) A two-loop finite solution, in which we keep $f^{\prime}$ non-vanishing and we use it to introduce the lepton masses. The model in turn becomes finite only up to two-loops since the corresponding solution of equation (7.4) is not an isolated one any more, i.e. it is a parametric one. In this case we have the following boundary conditions for the Yukawa couplings

$$
f^{2}=r\left(\frac{16}{9}\right) g^{2}, \quad f^{\prime 2}=(1-r)\left(\frac{8}{3}\right) g^{2},
$$

where $r$ is a free parameter which parametrizes the different solutions to the finiteness conditions. As for the boundary conditions of the soft scalars, we have the universal case.

\subsection{Predictions for $S U(3)^{3}$}

Below $M_{\mathrm{GUT}}$ all couplings and masses of the theory run according to the RGEs of the MSSM. Thus we examine the evolution of these parameters according to their RGEs up to two-loops for dimensionless parameters and at one-loop for dimensionful ones imposing the corresponding boundary conditions. We further assume a unique supersymmetry breaking scale $M_{\mathrm{SUSY}}$ and below that scale the effective theory is just the SM.

We compare our predictions with the most recent experimental value $m_{t}^{\exp }=(173.1 \pm$ 1.3) $\mathrm{GeV}$ [31], and recall that the theoretical values for $m_{t}$ suffer from a correction of $\sim 4 \%$ [20, $116,103]$. In the case of the bottom quark, we take again the value evaluated at $M_{Z}, m_{b}\left(M_{Z}\right)=$ $2.83 \pm 0.10 \mathrm{GeV}$ [115]. In the case of model I, the predictions for the top quark mass (in this case $m_{b}$ is an input) $m_{t}$ are $\sim 183 \mathrm{GeV}$ for $\mu<0$, which is above the experimental value, and there are no solutions for $\mu>0$.

For the two-loop model II, we look for the values of the parameter $r$ which comply with the experimental limits given above for top and bottom quarks masses. In the case of $\mu>0$, for the bottom quark, the values of $r$ lie in the range $0.15 \lesssim r \lesssim 0.32$. For the top mass, the range of values for $\mathrm{r}$ is $0.35 \lesssim r \lesssim 0.6$. From these values we can see that there is a very small region where both top and bottom quark masses are in the experimental range for the same value of $r$. In the case of $\mu<0$ the situation is similar, although slightly better, with the range of values $0.62 \lesssim r \lesssim 0.77$ for the bottom mass, and $0.4 \lesssim r \lesssim 0.62$ for the top quark mass. So far in the analysis, the masses of the new particles $h$ 's and E's of all families were taken to be at the $M_{\mathrm{GUT}}$ scale. Taking into account new thresholds for these exotic particles below $M_{\mathrm{GUT}}$ we hope to find a wider phenomenologically viable parameter space. The details of the predictions of the $S U(3)^{3}$ are currently under a careful re-analysis in view of the new value of the top-quark mass, the possible new thresholds for the exotic particles, as well as different intermediate gauge symmetry breaking into $S U(3)_{c} \times S U(2)_{L} \times S U(2)_{R} \times U(1)$ [142]. 


\section{Conclusions}

A number of proposals and ideas have matured with time and have survived after careful theoretical studies and confrontation with experimental data. These include part of the original GUTs ideas, mainly the unification of gauge couplings and, separately, the unification of the Yukawa couplings, a version of fixed point behaviour of couplings, and certainly the necessity of supersymmetry as a way to take care of the technical part of the hierarchy problem. On the other hand, a very serious theoretical problem, namely the presence of divergencies in Quantum Field Theories (QFT), although challenged by the founders of QFT [143, 144, 145], was mostly forgotten in the course of developments of the field partly due to the spectacular successes of renormalizable field theories, in particular of the SM. However, as it was already mentioned in the Introduction, fundamental developments in Theoretical Particle Physics are based in reconsiderations of the problem of divergencies and serious attempts to solve it. These include the motivation and construction of string and non-commutative theories, as well as $N=4$ supersymmetric field theories [3, 4], $N=8$ supergravity $[5,6,7,8,9]$ and the AdS/CFT correspondence [2]. It is a thoroughly fascinating fact that many interesting ideas that have survived various theoretical and phenomenological tests, as well as the solution to the UV divergencies problem, find a common ground in the framework of $N=1$ Finite Unified Theories, which we have described in the previous sections. From the theoretical side they solve the problem of UV divergencies in a minimal way. On the phenomenological side, since they are based on the principle of reduction of couplings (expressed via RGI relations among couplings and masses), they provide strict selection rules in choosing realistic models which lead to testable predictions. The celebrated success of predicting the top-quark mass [33, 34, 35, 36, 37, 38] is now extented to the predictions of the Higgs masses and the supersymmetric spectrum of the MSSM. At least the prediction of the lightest Higgs sector is expected to be tested in the next couple of years at LHC.

\section{Acknowledgements}

It is a pleasure for one of us (G.Z.) to thank the Organizing Committee for the very warm hospitality offered. This work is partially supported by the NTUA's basic research support programme 2008 and 2009, and the European Union's RTN programme under contract MRTN-

CT-2006-035505. Supported also by a mexican PAPIIT grant IN112709, and by Conacyt grants 82291 and 51554-F.

\section{References}

[1] Connes A., Douglas M.R., Schwarz A.S., Noncommutative geometry and matrix theory: compactification on tori, J. High Energy Phys. 1998 (1998), no. 2, 003, 35 pages, hep-th/9711162.

[2] Maldacena J.M., The large $N$ limit of superconformal field theories and supergravity, Adv. Theor. Math. Phys. 2 (1998), 231-252, hep-th/9711200.

[3] Mandelstam S., Light-cone superspace and the ultraviolet finiteness of the $N=4$ model, Nuclear Phys. B 213 (1983), 149-168.

[4] Brink L., Lindgren O., Nilsson B.E.W., The ultraviolet finiteness of the $N=4$ Yang-Mills theory, Phys. Lett. B 123 (1983), 323-328.

[5] Bern Z., Carrasco J.J., Dixon L.J., Johansson H., Roiban R., Ultraviolet behavior of $N=8$ supergravity at four loops, Phys. Rev. Lett. 103 (2009), 081301, 4 pages, arXiv:0905.2326.

[6] Kallosh R., On UV finiteness of the four loop $N=8$ supergravity, J. High Energy Phys. 2009 (2009), no. 9, 116, 9 pages, arXiv:0906.3495.

[7] Bern Z., Carrasco J.J., Dixon L.J., Johansson H., Kosower D.A., Roiban R., Cancellations beyond finiteness in $N=8$ supergravity at three loops, Phys. Rev. Lett. 98 (2007), 161303, 4 pages, hep-th/0702112. 
[8] Bern Z., Dixon L.J., Roiban R., Is $N=8$ supergravity ultraviolet finite?, Phys. Lett. B 644 (2007), 265-271, hep-th/0611086.

[9] Green M.B., Russo J.G., Vanhove P., Ultraviolet properties of maximal supergravity, Phys. Rev. Lett. 98 (2007), 131602, 4 pages, hep-th/0611273.

[10] Pati J.C., Salam A., Is baryon number conserved?, Phys. Rev. Lett. 31 (1973), 661-664.

[11] Georgi H., Glashow S.L., Unity of all elementary particle forces, Phys. Rev. Lett. 32 (1974), 438-441.

[12] Georgi H., Quinn H.R., Weinberg S., Hierarchy of interactions in unified gauge theories, Phys. Rev. Lett. 33 (1974), 451-454.

[13] Fritzsch H., Minkowski P., Unified interactions of leptons and hadrons, Ann. Physics 93 (1975), $193-266$.

[14] Georgi H., The state of the art - gauge theories, in Particles and Fields (Williamsburg, 1974), Editor C.E. Carlson, AIP Conference Proceedings, Vol. 23, American Institute of Physics, New York, 1974, 575-582.

[15] Amaldi U., de Boer W., Furstenau H., Comparison of grand unified theories with electroweak and strong coupling constants measured at LEP, Phys. Lett. B 260 (1991), 447-455.

[16] Dimopoulos S., Georgi H., Softly broken supersymmetry and SU(5), Nuclear Phys. B 193 (1981), 150-162.

[17] Sakai N., Naturalness in supersymmetric GUTs, Zeit. Phys. C 11 (1981), 153-157.

[18] Buras A.J., Ellis J.R., Gaillard M.K., Nanopoulos D.V., Aspects of the grand unification of strong, weak and electromagnetic interactions, Nuclear Phys. B 135 (1978), 66-92.

[19] Kubo J., Mondragón M., Olechowski M., Zoupanos G., Testing gauge-Yukawa-unified models by $M_{t}$, Nuclear Phys. B 479 (1996), 25-45, hep-ph/9512435.

[20] Kubo J., Mondragón M., Zoupanos G., Unification beyond GUTs: gauge Yukawa unification, Acta Phys. Polon. B 27 (1997), 3911-3944, hep-ph/9703289.

[21] Kobayashi T., Kubo J., Mondragón M., Zoupanos G., Exact finite and gauge-Yukawa unified theories and their predictions, Acta Phys. Polon. B 30 (1999), 2013-2027.

[22] Fayet P., Spontaneous generation of massive multiplets and central charges in extended supersymmetric theories, Nuclear Phys. B 149 (1979), 137-169.

[23] Decker R., Pestieau J., Lepton self-mass, Higgs scalar and heavy-quark masses, Nuovo Cimento Lett. 29 (1980), 560-564.

[24] Veltman M.J.G., The infrared-ultraviolet connection, Acta Phys. Polon. B 12 (1981), 437-457.

[25] Ferrara S., Girardello L., Palumbo F., A general mass formula in broken supersymmetry, Phys. Rev. D 20 (1979), 403-408.

[26] Chaichian M., Gonzalez Felipe R., Huitu K., On quadratic divergences and the Higgs mass, Phys. Lett. B 363 (1995), 101-105, hep-ph/9509223.

[27] Abbiendi G. et al., Search for the standard model Higgs boson at LEP, Phys. Lett. B 565 (2003), 61-75, hep-ex/0306033.

[28] Pendleton B., Ross G.G., Mass and mixing angle predictions from infrared fixed points, Phys. Lett. B 98 (1981), 291-294.

[29] Zimmermann W., Infrared behavior of the coupling parameters in the standard model, Phys. Lett. B 308 (1993), 117-122.

[30] Hill C.T., Quark and lepton masses from renormalization group fixed points, Phys. Rev. D 24 (1981), 691-703.

[31] Tevatron Electroweak Working Group, Combination of CDF and D0 Results on the mass of the top quark, arXiv:0903.2503.

[32] Schael S. et al., Search for neutral MSSM Higgs bosons at LEP, Eur. Phys. J. C 47 (2006), 547-587, hep-ex/0602042.

[33] Kapetanakis D., Mondragón M., Zoupanos G., Finite unified models, Z. Phys. C 60 (1993), 181-185, hep-ph/9210218.

[34] Mondragón M., Zoupanos G., Finite unified theories and the top quark mass, Nuclear Phys. B Proc. Suppl. 37 (1995), 98-105.

[35] Kubo J., Mondragón M., Zoupanos G., Reduction of couplings and heavy top quark in the minimal SUSY GUT, Nuclear Phys. B 424 (1994), 291-307. 
[36] Kubo J., Mondragón M., Tracas N.D., Zoupanos G., Gaug-Yukawa unification in asymptotically nonfree theories, Phys. Lett. B 342 (1995), 155-162, hep-th/9409003.

[37] Kubo J., Mondragón M., Shoda S., Zoupanos G., Gauge-Yukawa unification in SO(10) SUSY GUTs, Nuclear Phys. B 469 (1996), 3-20, hep-ph/9512258.

[38] Kubo J., Mondragón M., Zoupanos G., Perturbative unification of soft supersymmetry-breaking terms, Phys. Lett. B 389 (1996), 523-532, hep-ph/9609218.

[39] Zimmermann W., Reduction in the number of coupling parameters, Comm. Math. Phys. 97 (1985), 211-225.

[40] Oehme R., Zimmermann W., Relation between effective couplings for asymptotically free models, Comm. Math. Phys. 97 (1985), 569-582.

[41] Ma E., Modified quantum chromodynamics: exact global color symmetry and asymptotic freedom, Phys. Rev. D 17 (1978), 623-628.

[42] Ma E., Fixing the Higgs boson mass, Phys. Rev. D 31 (1985), 1143-1145.

[43] Lucchesi C., Piguet O., Sibold K., Necessary and sufficient conditions for all order vanishing $\beta$-functions in supersymmetric Yang-Mills theories, Phys. Lett. B 201 (1988), 241-244.

[44] Lucchesi C., Piguet O., Sibold K., Vanishing $\beta$-functions in $N=1$ supersymmetric gauge theories, Helv. Phys. Acta 61 (1988), 321-344.

[45] Lucchesi C., Zoupanos G., All-order finiteness in $N=1$ SYM theories: criteria and applications, Fortschr. Phys. 45 (1997), 129-143, hep-ph/9604216.

[46] Ermushev A.V., Kazakov D.I., Tarasov O.V., Finite $N=1$ supersymmetric grand unified theories, Nuclear Phys. B 281 (1987), 72-84.

[47] Kazakov D.I., Finite $N=1$ SUSY gauge field theories, Modern Phys. Lett. A 2 (1987), 663-674.

[48] Schrempp B., Schrempp F., A renormalization group invariant line and infrared attractive top-Higgs mass relation, Phys. Lett. B 299 (1993), 321-328.

[49] Schrempp B., Infrared fixed points and fixed lines in the top-bottom-tau sector in supersymmetric grand unification, Phys. Lett. B 344 (1995), 193-200, hep-ph/9411241.

[50] Schrempp B., Wimmer M., Top quark and Higgs boson masses: interplay between infrared and ultraviolet physics, Prog. Part. Nuclear Phys. 37 (1996), 1-90, hep-ph/9606386.

[51] Jack I., Jones D.R.T., Renormalization-group invariance and universal soft supersymmetry-breaking, Phys. Lett. B 349 (1995), 294-299, hep-ph/9501395.

[52] Hisano J., Shifman M.A., Exact results for soft supersymmetry-breaking parameters in supersymmetric gauge theories, Phys. Rev. D 56 (1997), 5475-5482, hep-ph/9705417.

[53] Jack I., Jones D.R.T., The gaugino $\beta$-function, Phys. Lett. B 415 (1997), 383-389, hep-ph/9709364.

[54] Avdeev L.V., Kazakov D.I., Kondrashuk I.N., Renormalizations in softly broken SUSY gauge theories, Nuclear Phys. B 510 (1998), 289-312, hep-ph/9709397.

[55] Kazakov D.I., Exploring softly broken SUSY theories via Grassmannian Taylor expansion, Phys. Lett. B 449 (1999), 201-206, hep-ph/9812513.

[56] Kazakov D.I., Finiteness of soft terms in finite $N=1$ SUSY gauge theories, Phys. Lett. B 421 (1998), 211-216, hep-ph/9709465.

[57] Jack I., Jones D.R.T., Pickering A., Renormalisation invariance and the soft $\beta$-functions, Phys. Lett. B 426 (1998), 73-77, hep-ph/9712542.

[58] Kobayashi T., Kubo J., Zoupanos G., Further all-loop results in softly-broken supersymmetric gauge theories, Phys. Lett. B 427 (1998), 291-299, hep-ph/9802267.

[59] Yamada Y., Two-loop renormalization group equations for soft supersymmetry-breaking scalar interactions: supergraph method, Phys. Rev. D 50 (1994), 3537-3545, hep-ph/9401241.

[60] Delbourgo R., Superfield perturbation theory and renormalization, Nuovo Cimento A 25 (1975), 646-656.

[61] Salam A., Strathdee J.A., Feynman rules for superfields, Nuclear Phys. B 86 (1975), 142-152.

[62] Fujikawa K., Lang W., Perturbation calculations for the scalar multiplet in a superfield formulation, Nuclear Phys. B 88 (1975), 61-76.

[63] Grisaru M.T., Siegel W., Rocek M., Improved methods for supergraphs, Nuclear Phys. B 159 (1979), 429-450.

[64] Girardello L., Grisaru M.T., Soft breaking of supersymmetry, Nuclear Phys. B 194 (1982), 65-76. 
[65] Jones D.R.T., Mezincescu L., Yao Y.P., Soft breaking of two loop finite $N=1$ supersymmetric gauge theories, Phys. Lett. B 148 (1984), 317-322.

[66] Jack I., Jones D.R.T., Soft supersymmetry breaking and finiteness, Phys. Lett. B 333 (1994), 372-379, hep-ph/9405233.

[67] Ibáñez L.E., Lüst D., Duality-anomaly cancellation, minimal string unification and the effective low-energy Lagrangian of 4D strings, Nuclear Phys. B 382 (1992), 305-364, hep-th/9202046.

[68] Kaplunovsky V.S., Louis J., Model-independent analysis of soft terms in effective supergravity and in string theory, Phys. Lett. B 306 (1993), 269-275, hep-th/9303040.

[69] Brignole A., Ibáñez L.E., Muñoz C., Towards a theory of soft terms for the supersymmetric standard model, Nuclear Phys. B 422 (1994), 125-171, hep-ph/9308271.

[70] Casas J.A., Lleyda A., Muñoz C., Problems for supersymmetry breaking by the dilaton in strings from charge and color breaking, Phys. Lett. B 380 (1996), 59-67, hep-ph/9601357.

[71] Kawamura Y., Kobayashi T., Kubo J., Soft scalar-mass sum rule in gauge-Yukawa unified models and its superstring interpretation, Phys. Lett. B 405 (1997), 64-70, hep-ph/9703320.

[72] Kobayashi T., Kubo J., Mondragón M., Zoupanos G., Constraints on finite soft supersymmetry-breaking terms, Nuclear Phys. B 511 (1998), 45-68, hep-ph/9707425.

[73] Novikov V.A., Shifman M.A., Vainshtein A.I., Zakharov V.I., Instanton effects in supersymmetric theories, Nuclear Phys. B 229 (1983), 407-420.

[74] Novikov V.A., Shifman M.A., Vainshtein A.I., Zakharov V.I., The beta function in supersymmetric gauge theories. Instantons versus traditional approach, Phys. Lett. B 166 (1986), 329-333.

[75] Shifman M.A., Little miracles of supersymmetric evolution of gauge couplings, Internat. J. Modern Phys. A 11 (1996), 5761-5784, hep-ph/9606281.

[76] Oehme R., Reduction and reparametrization of quantum field theories, Progr. Theoret. Phys. Suppl. (1986), no. $86,215-237$.

[77] Kubo J., Sibold K., Zimmermann W., Higgs and top mass from reduction of couplings, Nuclear Phys. B 259 (1985), 331-350.

[78] Kubo J., Sibold K., Zimmermann W., New results in the reduction of the standard model, Phys. Lett. B 220 (1989), 185-190.

[79] Piguet O., Sibold K., Reduction of couplings in the presence of parameters, Phys. Lett. B 229 (1989), 83-88.

[80] Zimmermann W., Reduction of couplings in massive models of quantum field theory, in Theoretical Physics. Fin de siècle (Wroclaw, 1998), Lecture Notes in Phys., Vol. 539, Springer, Berlin, 2000, 304-314.

[81] Wess J., Zumino B., A Lagrangian model invariant under supergauge transformations, Phys. Lett. B 49 (1974), 52-54.

[82] Iliopoulos J., Zumino B., Broken supergauge symmetry and renormalization, Nuclear Phys. B 76 (1974), $310-332$.

[83] Parkes A., West P.C., Finiteness in rigid supersymmetric theories, Phys. Lett. B 138 (1984), 99-104.

[84] Rajpoot S., Taylor J.G., On finite quantum field theories, Phys. Lett. B 147 (1984), 91-95.

[85] Rajpoot S., Taylor J.G., Toward finite quantum field theories, Int. J. Theor. Phys. 25 (1986), $117-138$.

[86] West P., The Yukawa $\beta$-function in $N=1$ rigid supersymmetric theories, Phys. Lett. B 137 (1984), 371-373.

[87] Jones D.R.T., Parkes A.J., Search for a three-loop-finite chiral theory, Phys. Lett. B 160 (1985), $267-270$.

[88] Jones D.R.T., Mezincescu L., The chiral anomaly and a class of two-loop finite supersymmetric gauge theories, Phys. Lett. B 138 (1984), 293-295.

[89] Parkes A.J., Three loop finiteness conditions in $N=1$ super-Yang-Mills, Phys. Lett. B 156 (1985), $73-79$.

[90] O'Raifeartaigh L., Spontaneous symmetry breaking for chiral scalar superfields, Nuclear Phys. B 96 (1975), $331-352$.

[91] Fayet P., Iliopoulos J., Spontaneously broken supergauge symmetries and goldstone spinors, Phys. Lett. B 51 (1974), 461-464.

[92] Ferrara S., Zumino B., Transformation properties of the supercurrent, Nuclear Phys. B 87 (1975), 207-220.

[93] Piguet O., Sibold K., The supercurrent in $N=1$ supersymmetrical Yang-Mills theories. I. The classical case, Nuclear Phys. B 196 (1982), 428-446. 
[94] Piguet O., Sibold K., The supercurrent in $N=1$ supersymmmetrical Yang-Mills theories. II. Renormalization, Nuclear Phys. B 196 (1982), 447-460.

[95] Piguet O., Sibold K., Nonrenormalization theorems of chiral anomalies and finiteness in supersymmetric Yang-Mills theories, Internat. J. Modern Phys. A 1 (1986), 913-942.

[96] Piguet O., Sibold K., Non-renormalization theorems of chiral anomalies and finiteness, Phys. Lett. B 177 (1986), 373-376.

[97] Ensign P., Mahanthappa K.T., The supercurrent and the Adler-Bardeen theorem in coupled supersymmetric Yang-Mills theories, Phys. Rev. D 36 (1987), 3148-3171.

[98] Piguet O., Supersymmetry, ultraviolet finiteness and grand unification, hep-th/9606045.

[99] Alvarez-Gaumé L., Ginsparg P.H., The topological meaning of non-abelian anomalies, Nuclear Phys. B 243 (1984), 449-474.

[100] Bardeen W.A., Zumino B., Consistent and covariant anomalies in gauge and gravitational theories, Nuclear Phys. B 244 (1984), 421-453.

[101] Zumino B., Wu Y.S., Zee A., Chiral anomalies, higher dimensions, and differential geometry, Nuclear Phys. B 239 (1984), 477-507.

[102] Leigh R.G., Strassler M.J., Exactly marginal operators and duality in four dimensional $N=1$ supersymmetric gauge theory, Nuclear Phys. B 447 (1995), 95-133, hep-th/9503121.

[103] Mondragón M., Zoupanos G., Higgs mass prediction in finite unified theories, Acta Phys. Polon. B 34 (2003), 5459-5468.

[104] Hamidi S., Patera J., Schwarz J.H., Chiral two-loop-finite supersymmetric theories, Phys. Lett. B 141 (1984), 349-352.

[105] Jiang X.D., Zhou X.J., Finite $N=1$ supersymmetric theories of classical groups, Phys. Lett. B 216 (1989), 160-166.

[106] Jiang X.D., Zhou X.J., Finite $N=1$ supersymmetric theories of $S U(N)$, Phys. Lett. B 197 (1987), 156-160.

[107] Jones D.R.T., Raby S., A two-loop finite supersymmetric $S U(5)$ theory: towards a theory of fermion masses, Phys. Lett. B 143 (1984), 137-141.

[108] León J., Pérez-Mercader J., Quirós M., Ramírez-Mittelbrunn J., A sensible finite SU(5) SUSY GUT?, Phys. Lett. B 156 (1985), 66-72.

[109] Kazakov D.I., Kalmykov M.Y., Kondrashuk I.N., Gladyshev A.V., Softly broken finite supersymmetric grand unified theory, Nuclear Phys. B 471 (1996), 389-408, hep-ph/9511419.

[110] Yoshioka K., Finite SUSY GUT revisited, Phys. Rev. D 61 (2000), 055008, 14 pages, hep-ph/9705449.

[111] Hamidi S., Schwarz J.H., A realistic finite unified theory?, Phys. Lett. B 147 (1984), 301-306.

[112] Babu K.S., Enkhbat T., Gogoladze I., Finite grand unified theories and the quark mixing matrix, Phys. Lett. B 555 (2003), 238-247, hep-ph/0204246.

[113] Heinemeyer S., Mondragón M., Zoupanos G., Confronting finite unified theories with low-energy phenomenology, J. High Energy Phys. 2008 (2008), no. 7, 135, 8 pages, arXiv:0712.3630.

[114] Carena M.S., Garcia D., Nierste U., Wagner C.E.M., Effective Lagrangian for the $\bar{t} b H^{+}$interaction in the MSSM and charged Higgs phenomenology, Nuclear Phys. B 577 (2000), 88-120, hep-ph/9912516.

[115] Amsler C. et al., Review of particle physics, Phys. Lett. B 667 (2008), 1-6.

[116] Kobayashi T., Kubo J., Mondragón M., Zoupanos G., Finite unification, Surveys High Energ. Phys. 16 (2001), 87-129.

[117] Barate R. et al., A measurement of the inclusive $b \rightarrow s \gamma$ branching ratio, Phys. Lett. B 429 (1998), $169-187$.

[118] Chen S. et al., Branching fraction and photon energy spectrum for $b \rightarrow s \gamma$, Phys. Rev. Lett. 87 (2001), 251807, 5 pages, hep-ex/0108032.

[119] Koppenburg P. et al., An inclusive measurement of the photon energy spectrum in $b \rightarrow s \gamma$ decays, Phys. Rev. Lett. 93 (2004), 061803, 6 pages, hep-ex/0403004.

[120] Heavy Flavor Analysis Group, see http://www.slac.stanford.edu/xorg/hfag/.

[121] Heinemeyer S., Hollik W., Weiglein G., FeynHiggs: a program for the calculation of the masses of the neutral $\mathcal{C}$ P-even Higgs bosons in the MSSM, Comput. Phys. Comm. 124 (2000), 76-89, hep-ph/9812320.

[122] Heinemeyer S., Hollik W., Weiglein G., The masses of the neutral $\mathcal{C} P$-even Higgs bosons in the MSSM: accurate analysis at the two-loop level, Eur. Phys. J. C 9 (1999), 343-366, hep-ph/9812472. 
[123] Degrassi G., Heinemeyer S., Hollik W., Slavich P., Weiglein G., Towards high-precision predictions for the MSSM Higgs sector, Eur. Phys. J. C 28 (2003), 133-143, hep-ph/0212020.

[124] Frank M. et al., The Higgs boson masses and mixings of the complex MSSM in the Feynman-diagrammatic approach, J. High Energy Phys. 2007 (2007), no. 2, 047, 56 pages, hep-ph/0611326.

[125] Davier M., Hoecker A., Malaescu B., Yuan C.Z., Zhang Z., Reevaluation of the hadronic contribution to the muon magnetic anomaly using new $e^{+} e^{-} \rightarrow \pi^{+} \pi^{-}$cross section data from BABAR, Eur. Phys. J. C 66 (2010), 1-9, arXiv:0908.4300.

[126] Goldberg H., Constraint on the photino mass from cosmology, Phys. Rev. Lett. 50 (1983), 1419-1422.

[127] Ellis J.R., Hagelin J.S., Nanopoulos D.V., Olive K.A., Srednicki M., Supersymmetric relics from the big bang, Nuclear Phys. B 238 (1984), 453-477.

[128] Valle J.W.F., Neutrino mass: theory, data and interpretation, hep-ph/9907222.

[129] Dreiner H.K., An introduction to explicit R-parity violation, Pramana 51 (1998), 123-133, hep-ph/9707435.

[130] Bhattacharyya G., A brief review of $R$-parity-violating couplings, hep-ph/9709395.

[131] Allanach B.C., Dedes A., Dreiner H.K., Bounds on R-parity violating couplings at the weak scale and at the GUT scale, Phys. Rev. D 60 (1999), 075014, 10 pages, hep-ph/9906209.

[132] Romão J.C., Valle J.W.F., Neutrino masses in supersymmetry with spontaneously broken $R$-parity, Nuclear Phys. B 381 (1992), 87-108.

[133] Lyth D.H., Stewart E.D., Thermal inflation and the moduli problem, Phys. Rev. D 53 (1996), 1784-1798, hep-ph/9510204.

[134] Gelmini G.B., Gondolo P., Neutralino with the right cold dark matter abundance in (almost) any supersymmetric model, Phys. Rev. D 74 (2006), 023510, 5 pages, hep-ph/0602230.

[135] Belanger G., Boudjema F., Pukhov A., Semenov A., micrOMEGAs: a program for calculating the relic density in the MSSM, Comput. Phys. Comm. 149 (2002), 103-120, hep-ph/0112278.

[136] Belanger G., Boudjema F., Pukhov A., Semenov A., MicrOMEGAs: Version 1.3, Comput. Phys. Comm. 174 (2006), 577-604, hep-ph/0405253.

[137] Ma E., Mondragón M., Zoupanos G., Finite $S U(N)^{k}$ unification, J. High Energy Phys. 2004 (2004), no. 12, 026, 17 pages, hep-ph/0407236.

[138] Glashow S.L., Trinification of all elementary particle forces, in Proceedings of Fifth Workshop on Grand Unification, Editors K. Kang, H. Fried and P. Frampton, World Scientific, Singapore, 1984, 88-94.

[139] Lazarides G., Panagiotakopoulos C., Shafi Q., Supersymmetric unification without proton decay, Phys. Lett. B 315 (1993), 325-330, hep-ph/9306332.

[140] Lazarides G., Panagiotakopoulos C., MSSM from SUSY trinification, Phys. Lett. B 336 (1994), 190-193, hep-ph/9403317.

[141] Ma E., Particle dichotomy and left-right decomposition of $E_{6}$ superstring models, Phys. Rev. D 36 (1987), 274-276.

[142] Heinemeyer S., Ma E., Mondragón M., Zoupanos G., Finite $S U(3)^{3}$ unification, in preparation.

[143] Dirac P.A.M., Lectures on quantum field theory, Academic Press Inc., 1967.

[144] Dyson F.J., Divergence of perturbation theory in quantum electrodynamics, Phys. Rev. 85 (1952), 631-632.

[145] Weinberg S., Living with infinities, arXiv:0903.0568 (and references therein). 